[Review]

\title{
基于 $\mathbf{g}-\mathrm{C}_{3} \mathrm{~N}_{4}$ 的 $\mathrm{Z}$ 型光催化体系研究进展
}

\author{
陈博才 沈 洋 魏建红 ${ }^{*}$ 熊 锐 石 兢 \\ (武汉大学物理科学与技术学院, 人工微纳结构教育部重点实验室, 武汉 430072)
}

\begin{abstract}
摘要: 导电聚合物型光催化材料 $\mathrm{g}-\mathrm{C}_{3} \mathrm{~N}_{4}$ 有着独特的电子结构、稳定的化学性能和显著的可见光催化活性。基 于 $g-C_{3} N_{4}$ 的 $Z$ 型光催化体系 $\left(Z-g-C_{3} N_{4}\right)$ 的催化效率高、电子-空穴复合率低而备受关注, 在光催化领域展现出 了巨大的应用潜力。本文阐述了 Z-g- $\mathrm{C}_{3} \mathrm{~N}_{4}$ 型光催化反应体系的作用机理, 综述了 Z-g- $\mathrm{C}_{3} \mathrm{~N}_{4}$ 在光催化领域的研 究进展, 介绍了 Z-g- $\mathrm{C}_{3} \mathrm{~N}_{4}$ 在产氢、转化 $\mathrm{CO}_{2}$ 、降解有机物等光催化领域的应用, 讨论了 $\mathrm{pH}$ 值、导电介质等因 素对 Z-g- $\mathrm{C}_{3} \mathrm{~N}_{4}$ 光催化性能的影响。最后指出了 Z-g- $\mathrm{C}_{3} \mathrm{~N}_{4}$ 光催化体系在研究过程中面临的问题和研究方向。
\end{abstract}

关键词: $\mathrm{g}-\mathrm{C}_{3} \mathrm{~N}_{4} ; \mathrm{Z}$ 型; 光催化体系; 电荷传输; 研究进展

中图分类号: 0643

\section{Research Progress on $\mathrm{g}-\mathrm{C}_{3} \mathrm{~N}_{4}$-Based Z-Scheme Photocatalytic System}

\author{
CHEN Bo-Cai SHEN Yang WEI Jian-Hong* XIONG Rui SHI Jing \\ (Key Laboratory of Artificial Micro- and Nano-structures of Ministry of Education, School of Physics and Technology, \\ Wuhan University, Wuhan 430072, P. R. China)
}

\begin{abstract}
Graphitic carbon nitride $\left(\mathrm{g}-\mathrm{C}_{3} \mathrm{~N}_{4}\right)$ is a promising photocatalyst because of its low cost, high stability, and visible-light-induced photocatalytic activity. Z-scheme photocatalysts based on g- $\mathrm{C}_{3} \mathrm{~N}_{4}\left(Z-g-\mathrm{C}_{3} \mathrm{~N}_{4}\right)$ have attracted considerable attention because of their lower recombination rate of electron-holes and higher catalytic efficiency. In this review, the reaction mechanism of Z-scheme photocatalysis and the recent progress in Z-g$\mathrm{C}_{3} \mathrm{~N}_{4}$ are introduced and reviewed. The applications of Z-g- $\mathrm{C}_{3} \mathrm{~N}_{4}$, such as water splitting and $\mathrm{CO}_{2}$ reduction, are presented. The key factors that affect the photocatalytic performance, such as $\mathrm{pH}$ and the presence of electron mediators, are discussed. Moreover, the current challenges are described and the future development of Z-g$\mathrm{C}_{3} \mathrm{~N}_{4}$ is forecast.
\end{abstract}

Key Words: $\quad$ g- $\mathrm{C}_{3} \mathrm{~N}_{4}$; Z-scheme; Photocatalytic system; Charge transfer; Research progress

\section{1 引言}

随着工业的飞速发展, 化石燃料(如煤、石 油、天然气等)被过度开采利用, 导致了严重的环 境污染和能源危机。据文献报道, 有限石油的储 存量将在今后的 50 年里消耗殆尽 1 。因此, 寻求一 种可以替代化石燃料的可再生能源迫在眉睫。太 阳能作为一种清洁能源, 取之不尽, 用之不竭,
是一种廉价可得的可再生能源 ${ }^{2}$, 开发利用太阳能 具有重要意义。氢能源具有高热值、燃烧无污染 等优点 ${ }^{3,4}$, 吸引了大量的科学研究者。半导体光催 化剂作为一种环境友好型材料, 利用太阳能制造 氢能源, 巧妙地将二者结合在一起, 有望解决工 业带来的环境问题和能源危机 ${ }^{5-8}$ 。光催化剂在光 解水产氢 ${ }^{9-11}$ 、还原 $\mathrm{CO}_{2}^{12-14}$ 、分解有害气体 ${ }^{15-17}$ 、降

Received: January 6, 2016; Revised: March 14, 2016; Published on Web: March 15, 2016.

${ }^{*}$ Corresponding author. Email: jhwei@whu.edu.cn; Tel: +86-27-68754613.

The project was supported by the National Natural Science Foundation of China (51272185) and National Key Basic Research Program of China (973) (2012CB821404). 
解有机物 ${ }^{18-20}$ 等方面有着巨大应用潜力。

迄今为止, $\mathrm{TiO}_{2}$ 被公认为是最有潜力的光催化 剂之一, 其光催化作用机理的研究也最为广泛 ${ }^{21}$ 。 1972 年 Fujishima 与 $\mathrm{Honda}^{22}$ 报道了 $\mathrm{TiO}_{2}$ 单晶电极光 解水产氢, 从此半导体光催化材料的研究步入新 的篇章。 $\mathrm{TiO}_{2}$ 化学稳定性好, 无毒无害, 但其电 子-空穴复合率高, 能带间隙较宽, 仅能响应紫外 区域的波段 ${ }^{23,24}$, 严重限制了其应用范围。近年 来, 一种新型的可见光响应半导体光催化材料 $\mathrm{g}$ $\mathrm{C}_{3} \mathrm{~N}_{4}$ 受到了广泛的关注 ${ }^{25-27}$ 。2009 年 Wang 等 ${ }^{28}$ 首次 报道了 $\mathrm{g}-\mathrm{C}_{3} \mathrm{~N}_{4}$ 在可见光区域光解水产氢, 此后大量 文献对 $g-C_{3} N_{4}$ 体系的研究进行了报道。g- $\mathrm{C}_{3} \mathrm{~N}_{4}$ 的合 成方法简单、制备成本低, 三聚氭胺、二氧二 胺、尿素等原料仅通过简单的炦烧就可制得催化 性能较好的 $\mathrm{g}-\mathrm{C}_{3} \mathrm{~N}_{4}{ }^{29-31}$ 。g- $\mathrm{C}_{3} \mathrm{~N}_{4}$ 能隙位置独特, 能 够在可见光下完成对半导体要求较高的光催化反 应, 吸引了大量的研究者。但由于 $\mathrm{g}-\mathrm{C}_{3} \mathrm{~N}_{4}$ 激子结合 能高、结晶度低, 使得光生电子-空穴对难以分 离, 光生载流子迁移率小, 导致光催化过程量子 效率偏低。科学工作者们对 $\mathrm{g}-\mathrm{C}_{3} \mathrm{~N}_{4}$ 进行了大量的改 性研究, Wang 等 ${ }^{32}$ 以 $\mathrm{BmimBF}_{4}$ 和二氰二胺为原料

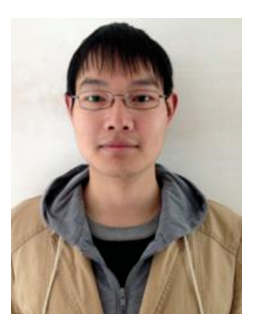

陈博才, 武汉大学物理科学与技术 学院, 硕士研究生。研究方向: 半 导体光催化材料的改性研究。
合成了 $B 、 F$ 共掺型 $g-C_{3} N_{4}$, 扩宽了 $g-C_{3} N_{4}$ 在可见 光区域的吸收范围, 增强了其在紫外-可见光区域 的光吸收能力, 提高了其对有机物选择性氧化的 几率。Zhang 等 ${ }^{33}$ 采用被烧法将石墨烯插入 $\mathrm{g}-\mathrm{C}_{3} \mathrm{~N}_{4}$ 层间, 石墨烯与 $\mathrm{g}-\mathrm{C}_{3} \mathrm{~N}_{4}$ 之间的 $\pi-\pi$ 作用, 大幅度提 升了材料的光电性能。 $\mathrm{Liu}$ 课题组 ${ }^{34}$ 在 $450{ }^{\circ} \mathrm{C}$ 条件 下, 将 $\mathrm{H}_{2} \mathrm{~S}$ 气流通入载有 $\mathrm{g}-\mathrm{C}_{3} \mathrm{~N}_{4}$ 的管式炉中, 制备 出了硫掺杂 $\mathrm{g}-\mathrm{C}_{3} \mathrm{~N}_{4}$ 。掺硫后 $\mathrm{g}-\mathrm{C}_{3} \mathrm{~N}_{4}$ 价带位置升高, 带隙宽度变窄, 在一定范围的光照条件下, 其产 氢性能远优于未改性的样品。大量的改性研究使 得 $g-\mathrm{C}_{3} \mathrm{~N}_{4}$ 光催化剂的性能有了较大的提升, 但其可 见光利用范围依然有很大的拓展空间, 仅通过掺 杂改性也无法解决 $\mathrm{g}-\mathrm{C}_{3} \mathrm{~N}_{4}$ 自身电子-空穴容易复合 的问题。克服半导体的自身因素限制问题, 必须 从两方面着手: 一方面, 减小半导体的禁带宽 度, 可以扩宽光谱响应范围。另一方面, 使导带 电势更负, 价带电势更正, 在热力学上更有利于 光催化体系中的氧化还原反应 ${ }^{35}$ 。然而这两点是相 悖的, 第一点要求带隙变窄; 第二点中导带电势 更负, 价带电势更正会使得带隙变宽。对于单组 分的光催化剂而言, 不可能同时做到以上两点, 新型人工 $Z$ 型光催化材料, 由多组分构成, 能同时 满足以上两点要求。

$\mathrm{Z}$ 型光催化反应体系因其电荷传输机理类似于 自然界中绿色植物的光合作用而得名 ${ }^{36}$, 由两个光 反应系统组成, 光反应系统 I (photosystem I, 记为 PS I) 和光反应系统 II (photosystem II, 记为 PS II)。 $\mathrm{Z}$ 型光催化剂以导电介质在溶液中的存在状态又可

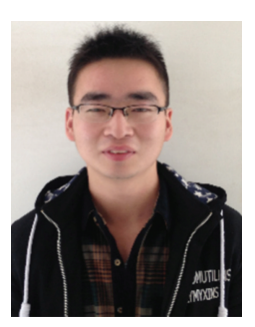

沈洋, 武汉大学物理科学与技术学 院, 硕士研究生。研究方向: 半导 体光催化材料在污水净化方面的应 用。

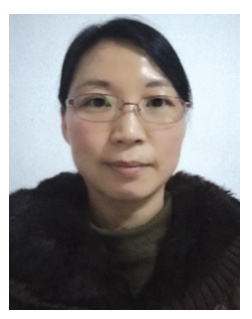

魏建红, 武汉大学物理科学与技术 学院副教授, 博士生导师。主要从 事有机-无机纳米复合材料、光催 化材料、电磁流变材料及材料的腐 蚀与防护性能研究。

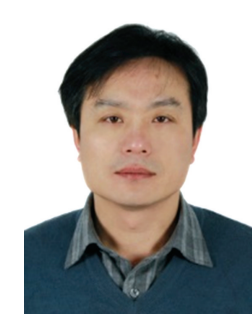

熊锐, 武汉大学物理科学与技术学 院教授, 博士生导师。主要从事无 机、有机半导体材料及其掺杂体系 的光催化及稀磁特性; 双钙钛矿结 构材料的高介电性、电荷分布及电 荷转移性能研究等。

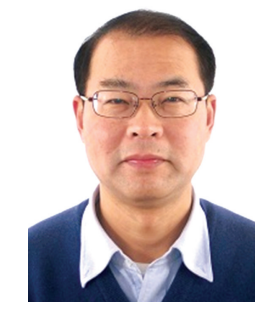

石兢, 武汉大学物理科学与技术 学院教授, 博士生导师。主要研 究方向: 能源材料中高效热电材 料的设计及性能优化, 光催化材 料及强场电绝缘材料的老化与击 穿机理研究等。 
分为两类, 离子态 $\mathrm{Z}$ 型光催化剂和固态 $\mathrm{Z}$ 型光催化 剂, 不含导电介质的体系归纳为固态类。 $Z$ 型光催 化体系研究历程中, 由于氧化还原电子介体缺陷 的缘故, 离子态 $\mathrm{Z}$ 型光催化剂逐渐被固态 $\mathrm{Z}$ 型光催 化剂所取代。其独特的能隙结构和电荷传输方式 使得电子与空穴在空间上分离开来, 光催化过程 中电子空穴复合率低 ${ }^{37}$, 表现出强氧化还原性, 吸 引了大量的科学研究者。 $\mathrm{g}-\mathrm{C}_{3} \mathrm{~N}_{4}$ 与另一光催化剂复 合构成 $\mathrm{Z}$ 型结构后, 克服了 $\mathrm{g}-\mathrm{C}_{3} \mathrm{~N}_{4}$ 原本存在的电 子-空穴复合率高、循环稳定性能差等问题, 表现 出了更加优异的光催化性能。Z-g- $\mathrm{C}_{3} \mathrm{~N}_{4}$ 是固态 $\mathrm{Z}$ 型 光催化剂中一类典型代表, 该体系的光催化剂在 光解水产氢、还原 $\mathrm{CO}_{2}$ 、分解有机物等方面表现出 了优异的光催化性能。近年来大量的文献报道了 $\mathrm{Z}-\mathrm{g}-\mathrm{C}_{3} \mathrm{~N}_{4}$ 光催化体系 ${ }^{38,39}$, 本文着重介绍了 Z 型光催 化反应体系的机理以及 $\mathrm{Z}-\mathrm{g}-\mathrm{C}_{3} \mathrm{~N}_{4}$ 光催化剂在光解 水、还原 $\mathrm{CO}_{2}$ 等方面的应用, 并讨论了 $\mathrm{pH}$ 值、结 构等因数对光催化性能的影响, 指出了该领域今 后可能的研究方向。

\section{$2 Z$ 型光催化反应体系的机理}

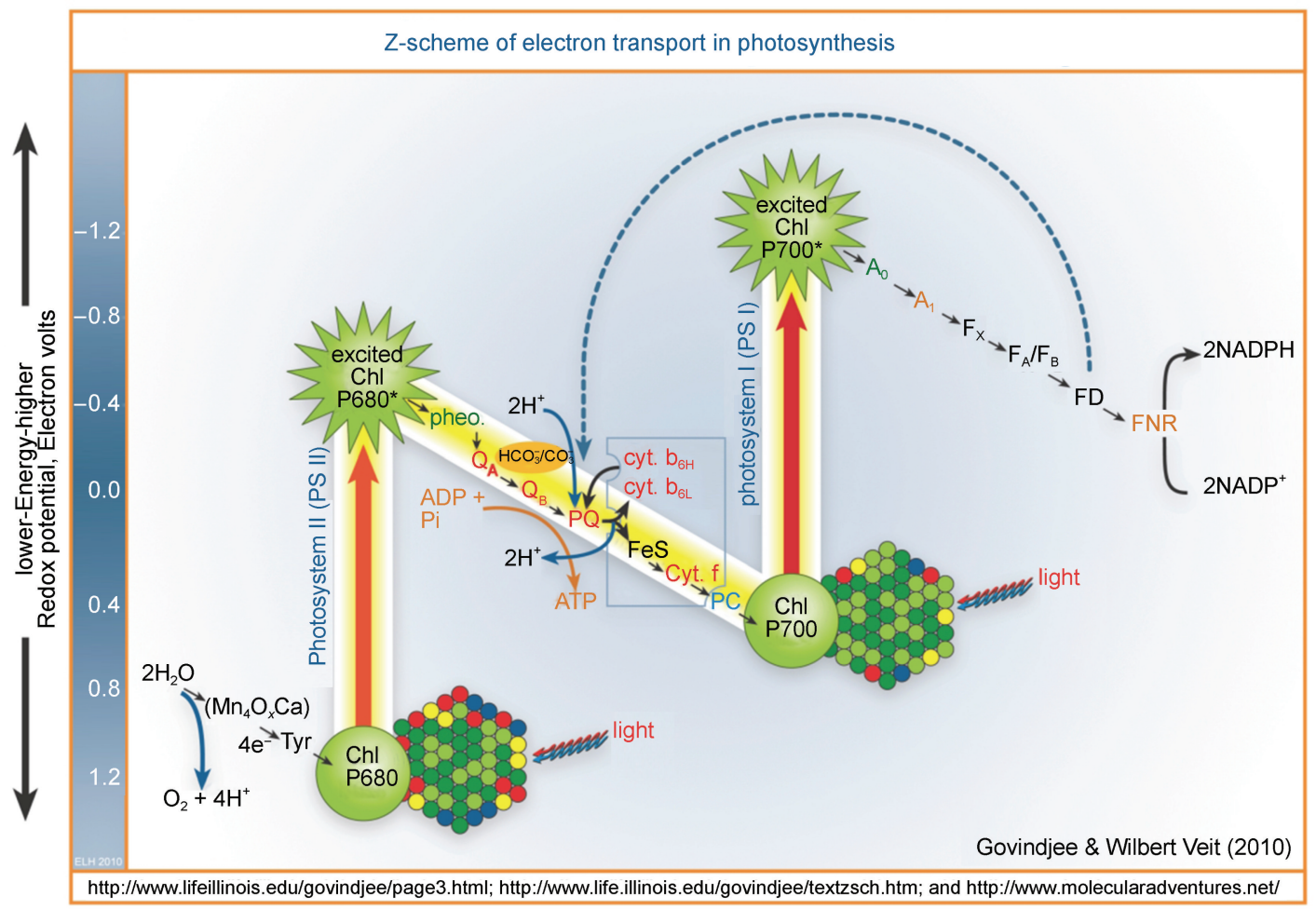

图 1 植物光合作用过程中电子的 “ $Z$ 型” 传递机理图 ${ }^{42}$

Fig.1 Z-scheme of electron transport mechanism in plant photosynthesis progress ${ }^{42}$ ADP: adenosine diphosphate; ATP: adenosine triphosphate; PC: plastocyanin; $\mathrm{A}_{0}$ : phylloquinone; $\mathrm{A}_{1}$ : vitamin $\mathrm{K}_{1}$; $\mathrm{F}_{\mathrm{X}}, \mathrm{F}_{\mathrm{A}} / \mathrm{F}_{\mathrm{B}}$ : primary electron acceptor of PS I; FD: ferredoxin; $\mathrm{NADP}^{+}$: nicotinamide adenine dinucleotide phosphate 


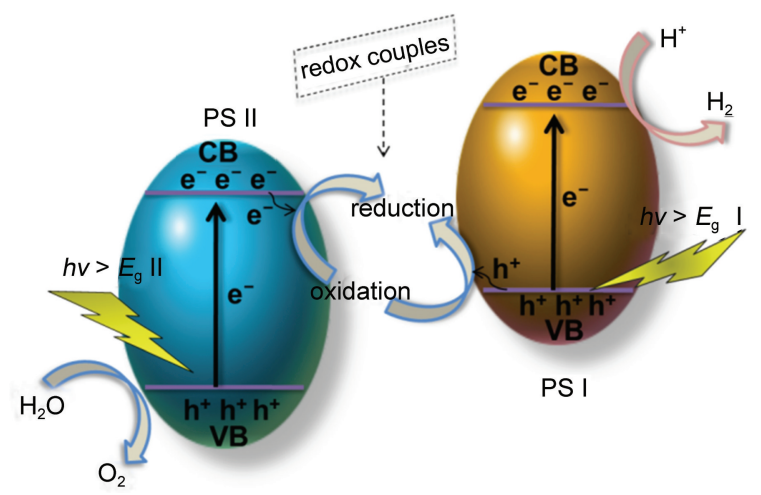

图 2 离子态 $\mathrm{Z}$ 型光催化反应电荷传递示意图

Fig.2 Electron transfer schematic diagram for ionic-state Z-scheme in photocatalytic reaction

$\mathrm{CB}$ : conduction band; VB: valence band

$\mathrm{IO}_{3}^{-} / \mathrm{I}^{-45,46} 、\left[\mathrm{Co}(\mathrm{bpy})_{3}\right]^{3+2+}$ 和 $\left[\mathrm{Co}(\text { phen })_{3}\right]^{3+2+47}$ 。离子态 $\mathrm{Z}$ 型光催化反应的电荷传递机理如图 2 所示。PS II 的价带受到光激发, 电子跃迁至导带处, PS II 价 带处的空穴可以将 $\mathrm{H}_{2} \mathrm{O}$ 氧化为 $\mathrm{O}_{2}$, 并产生氢离 子。PS II 导带处的激发态电子与氧化还原电子介 体中的高价态离子反应, 而被消耗。PS I 的价带受 到光激发, 电子跃迁到导带处将 $\mathrm{H}^{+}$还原为氢气。 PS I 价带的空穴与氧化还原电子介体中的低价态 离子反应生成高价态离子。以 $\left[\mathrm{Co}(\mathrm{phen})_{3}\right]^{3+2+}$ 为 例, PS II 导带处激发态电子与 $\left[\mathrm{Co}(\mathrm{phen})_{3}\right]^{3+}$ 反应生 成 $\left[\mathrm{Co}(\mathrm{phen})_{3}\right]^{2+}$, PS I 的价带空穴与 $\left[\mathrm{Co}(\mathrm{phen})_{3}\right]^{2+}$ 反 应生成 $\left[\mathrm{Co}(\mathrm{phen})_{3}\right]^{3+47}$ 。可以用如下方程进行表述:

$$
\begin{aligned}
& {\left[\mathrm{Co}(\text { phon })_{3}\right]^{3+}+\mathrm{e}_{\mathrm{CB}}^{-}(\text {PS II }) \stackrel{h v}{\rightarrow}\left[\mathrm{Co}(\text { phon })_{3}\right]^{2+}} \\
& {\left[\mathrm{Co}(\text { phon })_{3}\right]^{2+}+\mathrm{h}_{\mathrm{VB}}^{+}(\text {PS I }) \stackrel{h v}{\rightarrow}\left[\mathrm{Co}(\text { phon })_{3}\right]^{3+}}
\end{aligned}
$$

固态 $\mathrm{Z}$ 型光催化体系与离子态 $\mathrm{Z}$ 型光催化体系 的催化机理又有所差异, 固态 $Z$ 型光催化体系不含 氧化还原电子介体, 电荷直接通过界面传输, 缩 短了传输距离, 提高了光催化效率。固态 $\mathrm{Z}$ 型光催
化剂可分为两类(图 3), 无导电介质的光催化剂(图 3(a)) 和有导电介质的光催化剂(图 3(b))。如图 3(b) 所示, 在光照条件下, PS I 和 PS II 的价带留下光 生空穴, 电子被激发到 PS I 和 PS II 的导带上。PS II 价带上的光生空穴有很强的氧化能力, 能够氧化 水、有机污染物等 ${ }^{48,49}$ 。PS II 导带上的电子与 PS I 价带的空穴在导电介质上湮灭。PS I 导带处的激发 态电子有很强的还原能力, 能产氢, 还原 $\mathrm{CO}_{2}$ 等 ${ }^{50,51}$ 。无导电介质的 $Z$ 型光催化剂电荷传输机理 和上述相似。 $\mathrm{Z}$ 型光催化材料较之传统的异质结纳 米复合光催化材料往往具有更强的氧化还原能 力。这是因为异质结型光催化材料的氧化反应和 还原反应分别发生在 PS I 和 PS II 的价带及导带 上, 虽有利于光生电子和空穴的分离, 但 PS I 的价 带位置相对于 PS II 靠上, 氧化能力减弱; PS II 的 导带位置相对于 PS I靠下, 还原能力减弱。因此, 异质结光催化材料相对于 PS I、PS II 随拓宽了它 们的光响应范围, 但导致它们相应的氧化、还原 能力下降。复合 Z 型光催化材料既能保证宽的光响 应范围, 又能提高 PS I 及 PS II 的氧化、还原能 力, 因而显示出更强的应用前景。

Z 型光催化体系对 PS I 和 PS II 的能隙有一定 要求, PS II 的导带电势必须要比 PS I 的价带电势 低，两者的导带、价带电势要有一定范围的落 差。PS II 的价带电势越正, Z 型光催化体系的氧化 能力越强; PS I 的导带电势越负, Z 型光催化体系 的还原能力越强。

随着人们对 $Z$ 型光催化反应的进一步了解, 离 子态 $\mathrm{Z}$ 型光催化反应体系逐渐被取代, 这是由于其 如下几个弊端造成: (a) 容易发生副反应, 氧化还 原电子介体中的高价态离子容易与 PS I 的导带电子



(a)

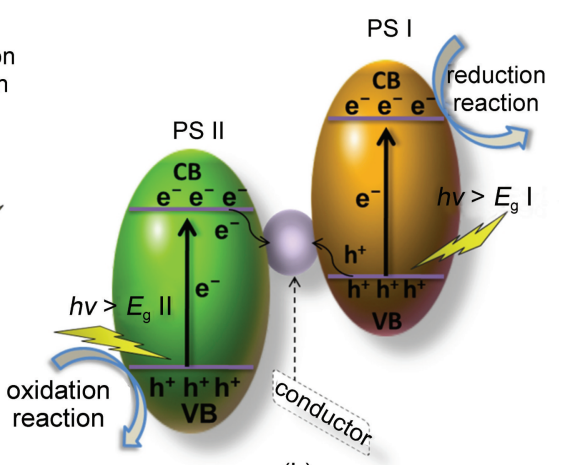

(b)

图 3 全固态 $\mathrm{Z}$ 型光催化反应电荷传递示意图

Fig.3 Electron transfer schematic diagram for all-solid-state Z-scheme photocatalytic reaction (a) no electron mediator in the system; (b) with electron mediator in the system 
发生反应, 同理, 氧化还原电子介体中的低价态 离子也会发生类似的发应 ${ }^{52}$; (b) 半导体光吸收减 少, 氧化还原电子介体吸收了部分入射光; (c) 应 用范围狭窄, 仅限于光解水一类 ${ }^{52,53}$; (d) 不能按比 例产氢和产氧 ${ }^{54}$; (e) 光解水时逆反应程度高。随 着研究的深入, 人们开发了不含氧化还原电子介 体的固态 $\mathrm{Z}$ 型光催化剂, 固态 $\mathrm{Z}$ 型光催化体系能很 好的克服上述离子态 $Z$ 型光催化反应中存在的大多 数的问题。在固态 $Z$ 型光催化反应体系中, 电荷直 接通过界面接触传输, 缩短了传输距离, 降低了 副反应的发生几率, 提高了可见光利用率和光催 化效率, 其应用领域不仅仅限于光解水。故而, 离子态 $Z$ 型光催化剂逐渐被固态 $Z$ 型光催化剂所取 代。在 Z-g- $\mathrm{C}_{3} \mathrm{~N}_{4}$ 体系中, 由于 $\mathrm{g}-\mathrm{C}_{3} \mathrm{~N}_{4}$ 导带价带位置 相对较高, PS I组分一般由 $\mathrm{g}-\mathrm{C}_{3} \mathrm{~N}_{4}$ 构成。Z-g- $\mathrm{C}_{3} \mathrm{~N}_{4}$ 的电荷传递机理与上述固态 $Z$ 型光催化体系电荷传 递机理类似, 在下部分会用实例进行详细的介绍。

\section{Z-g- $\mathrm{C}_{3} \mathrm{~N}_{4}$ 光催化剂的应用}

$\mathrm{Z}-\mathrm{g}-\mathrm{C}_{3} \mathrm{~N}_{4}$ 光催化剂在光解水、还原 $\mathrm{CO}_{2}$ 、分解 有害气体、降解有机物等方面有着优异的光催化 性能和广阔的商业应用前景, 大量的文献报道了 $\mathrm{Z}-\mathrm{g}-\mathrm{C}_{3} \mathrm{~N}_{4}$ 在光催化领域的应用。基于篇幅限制, 表 1 仅列举了部分 $Z-g-C_{3} N_{4}$ 光催化体系在催化领域的 应用 ${ }^{39,55-76}$ 。

\section{1 光分解水}

氢气是清洁能源, 燃烧过程中不产生有毒有 害气体, 有很高的能量密度 ${ }^{76-78}$ 。因此, 氢能源有 望取代化石燃料成为下一代核心能源 ${ }^{79}$ 。当前, 氢 气的主流制备途径是传统的碳氢化合物反应法 ${ }^{80}$, 这类制备方法消耗了大量的能量, 排出了大量的 废气, 如 $\mathrm{CO}_{2}$ 和 $\mathrm{CO}$ 等, 无法做到清洁、可持续性 利用。半导体光催化剂在水溶液条件下, 太阳能 光照产氢, 清洁无污染, 受到了广大研究者的青 棟。Z-g- $\mathrm{C}_{3} \mathrm{~N}_{4}$ 光催化剂在光解水产氢中表现出了优 异的性能。

Z-g- $\mathrm{C}_{3} \mathrm{~N}_{4}$ 光催化体系中, 光分解水产氢和产氧 分别发生在 PS I 的导带和 PS II 的价带上, 可以由 如下三个光化学方程进行表述。

光生电子在 PS I 的导带上将 $\mathrm{H}^{+}$还原为 $\mathrm{H}_{2}$ ：

$$
2 \mathrm{H}^{+}+2 \mathrm{e}^{-} \stackrel{h v}{\rightarrow} \mathrm{H}_{2}
$$

光生空穴在 PS II 的价带上氧化 $\mathrm{H}_{2} \mathrm{O}$ 产生 $\mathrm{O}_{2}$ :

$$
\mathrm{H}_{2} \mathrm{O}+2 \mathrm{~h}^{+} \stackrel{h v}{\rightarrow} \frac{1}{2} \mathrm{O}_{2}+2 \mathrm{H}^{+}
$$

总反应:

$$
\mathrm{H}_{2} \mathrm{O} \stackrel{h v}{\rightarrow} \frac{1}{2} \mathrm{O}_{2}+\mathrm{H}_{2}
$$

水分解反应属于 “上坡反应”, 在水分解的过 程中标准吉布斯自由能正向变化 $+237.46 \mathrm{~kJ}$ $\mathrm{mol}^{-181}$, 使得反应不能自发进行。Z-g- $\mathrm{C}_{3} \mathrm{~N}_{4}$ 光催化 体系将反应分为两步, 每一步需要的能量较总反 应能量小得多, 使得反应更容易发生。Katsumata 等 ${ }^{82}$ 通过简单的焙烧法制备出了无导电介质的 Z型 光催化剂 $\mathrm{WO}_{3} / \mathrm{g}-\mathrm{C}_{3} \mathrm{~N}_{4}, \mathrm{WO}_{3}$ 颗粒分散在光滑的 $\mathrm{g}-$ $\mathrm{C}_{3} \mathrm{~N}_{4}$ 表面。 $\mathrm{WO}_{3}(10 \%(w)) / \mathrm{g}-\mathrm{C}_{3} \mathrm{~N}_{4}$ 的光致发光光谱 在 $450 \mathrm{~nm}$ 处峰值明显比 $\mathrm{g}-\mathrm{C}_{3} \mathrm{~N}_{4}$ 的低, 表明形成 $\mathrm{Z}$ 型结构后, 光生电子和空穴在 $\mathrm{g}-\mathrm{C}_{3} \mathrm{~N}_{4}$ 表面的复合率 降低。电子空穴复合率的结果与产氢的结果一 致, $\mathrm{WO}_{3}(10 \%(w)) / \mathrm{g}-\mathrm{C}_{3} \mathrm{~N}_{4}$ 在可见光下的产氢速率 为 $66 \mu \mathrm{mol} \cdot \mathrm{h}^{-1} \cdot \mathrm{g}^{-1}$, 而纯 $\mathrm{g}-\mathrm{C}_{3} \mathrm{~N}_{4}$ 的产氢速率只有 $27 \mu \mathrm{mol} \cdot \mathrm{h}^{-1} \cdot \mathrm{g}^{-1}$ 。 $\mathrm{Z}$ 型结构的 $\mathrm{WO}_{3}(10 \%(w)) / \mathrm{g}-\mathrm{C}_{3} \mathrm{~N}_{4}$ 在循环实验中也表现出了更强的稳定性。

与上述无导电介质光催化剂相比, 有导电介 质的 Z-g- $\mathrm{C}_{3} \mathrm{~N}_{4}$ 光催化剂同样表现出了优异的光催化 性能。 $\mathrm{Li}$ 等 ${ }^{74}$ 采用三步法合成了 $\mathrm{g}-\mathrm{CNS} / \mathrm{Au} / \mathrm{CdS}$ 的 $Z$ 型光催化剂(氮掺杂 $g-C_{3} \mathrm{~N}_{4}$ 记为 $g-C N S$ ), 在可见 光照射下, $\mathrm{CdS}$ 导带上的光生电子转移至 $\mathrm{Au}$ 上, 与此同时 $\mathrm{g}-\mathrm{CNS}$ 价带上的光生空穴传递至 $\mathrm{Au}$ 与光 生电子发生湮灭。g-CNS 导带上的电子表现出强还 原能力, 将 $\mathrm{H}^{+}$还原为 $\mathrm{H}_{2}$ 。纯 $\mathrm{g}-\mathrm{CNS}$ 和 $\mathrm{CdS}$ 在可见 光照射下, $5 \mathrm{~h}$ 的氢气产量分别为 15.4 和 126.5 $\mu \mathrm{mol}$ 。两者复合加入导电介质构成 Z 型的光催化剂 后, $5 \mathrm{~h}$ 可见光下氢气的产量高达 $530 \mu \mathrm{mol}, \mathrm{Z}$ 型 结构大幅度提升了半导体光催化剂的光解水性能。

目前已有大量文献报道了 $\mathrm{Z}-\mathrm{g}-\mathrm{C}_{3} \mathrm{~N}_{4}$ 光催化剂在 模拟可见光或太阳光条件下光解水, 如 $\mathrm{Z}$ 型 $\mathrm{WO}_{3}$ $\mathrm{C}_{3} \mathrm{~N}_{4}$ 复合体系 ${ }^{39,69}, \mathrm{AgPO}_{4}-\mathrm{C}_{3} \mathrm{~N}_{4}$ 复合体系 ${ }^{75,83}$ 。人们 对该体系光解水机理的研究也越来越深入。

\section{2 分解有害气体、降解有机物}

\subsection{1 分解有害气体}

随着人们对生活品质的要求不断提高, 空气 污染越来越严重(装修污染、建筑污染、汽车尾气 排放等等), 导致了各种疾病的高频产生 ${ }^{84,85}$, 半导 体光催化剂在空气净化方面有着广泛的应用 ${ }^{86}$ 。甲 醛是典型的室内有害气体, 容易引发皮肤病、呼 吸道疾病、白血病等 ${ }^{87}$, 但房屋装修不可避免地会 产生大量的甲醛。 $\mathrm{Yu}$ 等 ${ }^{55}$ 合成了 $\mathrm{Z}$ 型 $\mathrm{g}-\mathrm{C}_{3} \mathrm{~N}_{4} / \mathrm{TiO}_{2}$ 光 
表 1 目前报道的部分 $\mathrm{Z}-\mathrm{g}-\mathrm{C}_{3} \mathrm{~N}_{4}$ 在光催化领域的应用

Table 1 Summary of the application of $\mathrm{Z}-\mathrm{g}-\mathrm{C}_{3} \mathrm{~N}_{4}$ in the photocatalytic field so far

\begin{tabular}{|c|c|c|c|c|c|c|}
\hline $\begin{array}{l}\text { PS I (available } \\
\text { wavelength/nm) }\end{array}$ & $\begin{array}{l}\text { PS II (available } \\
\text { wavelength/nm) }\end{array}$ & $\begin{array}{c}\text { Light source } \\
\text { (wavelength/nm) }\end{array}$ & $\begin{array}{l}\text { Electron } \\
\text { mediator }\end{array}$ & Application & Activity & Ref. \\
\hline $\mathrm{g}-\mathrm{C}_{3} \mathrm{~N}_{4}(<460)$ & $\mathrm{TiO}_{2}(<387)$ & 15 W UV lamp 365 & & $\mathrm{De}^{\mathrm{a}}(\mathrm{HCHO})$ & $\eta_{\mathrm{HCHO}}^{\mathrm{b}}=94 \%(1 \mathrm{~h})$ & 55 \\
\hline $\mathrm{g}-\mathrm{C}_{3} \mathrm{~N}_{4}(<460)$ & $\mathrm{Ti}^{3+} / \mathrm{TiO}_{2}(<410)$ & $11 \mathrm{~W} \mathrm{INC}^{\mathrm{c}}$ lamp (420-800) & & De (phenol) & $\eta_{\text {phenol }}=74 \%(7 \mathrm{~h})$ & 56 \\
\hline $\mathrm{g}-\mathrm{C}_{3} \mathrm{~N}_{4}(<464)$ & $\mathrm{WO}_{3}(<461)$ & LED (ca 435) & & De $\left(\mathrm{C}_{2} \mathrm{H}_{5} \mathrm{OH}\right)$ & $\begin{array}{l}\text { at least } 600 \times 10^{-6} \\
\text { acetaldehyde }(48 \mathrm{~h})\end{array}$ & 39 \\
\hline $\mathrm{g}-\mathrm{C}_{3} \mathrm{~N}_{4}(<460)$ & $\mathrm{Ag}_{3} \mathrm{PO}_{4}(<530)$ & 300 W Xe lamp (300-800) & & De (ethylene) & $\eta_{\mathrm{C} 2 \mathrm{H} 4} \approx 100 \%(3 \mathrm{~h})$ & 57 \\
\hline $\mathrm{g}-\mathrm{C}_{3} \mathrm{~N}_{4}(<460)$ & $\mathrm{ZnO}(<387)$ & 300 W Xe-arc lamp (300-800) & & $\mathrm{CO}_{2}$ reduction & $\begin{array}{c}\mathrm{CH}_{3} \mathrm{OH} \text { production rate } \\
0.6 \mu \mathrm{mol} \cdot \mathrm{h}^{-1} \cdot \mathrm{g}^{-1}\end{array}$ & 58 \\
\hline $\mathrm{g}-\mathrm{C}_{3} \mathrm{~N}_{4}(<464)$ & $\mathrm{V}_{2} \mathrm{O}_{5}(<558)$ & $250 \mathrm{~W}$ Xe lamp $(>420)$ & & $\mathrm{De}\left(\mathrm{TC}^{\mathrm{d}}\right)$ & $\eta_{\mathrm{TC}}=95.5 \%(1 \mathrm{~h})$ & 59 \\
\hline $\mathrm{g}-\mathrm{C}_{3} \mathrm{~N}_{4}(<460)$ & $\operatorname{AgBr}(<550)$ & 300 W Xe lamp (400-680) & $\mathrm{Ag}$ & $\operatorname{De}\left(\mathrm{RhB}^{\mathrm{e}}, \mathrm{MO}^{\mathrm{f}}\right)$ & $\begin{array}{l}\eta_{\mathrm{RhB}}=95 \%(10 \mathrm{~min}) \\
\eta_{\mathrm{MO}}=95 \%(10 \mathrm{~min})\end{array}$ & 60 \\
\hline g- $\mathrm{C}_{3} \mathrm{~N}_{4}(<460)$ & $\mathrm{Bi}_{2} \mathrm{O}_{3}(<442)$ & 500 W Xe lamp (400-470) & & $\mathrm{De}\left(\mathrm{MB}^{\mathrm{g}}, \mathrm{RhB}\right)$ & $\begin{array}{l}\eta_{\mathrm{MB}}=96 \%(2 \mathrm{~h})^{*} \\
\eta_{\mathrm{RhB}}=70 \%(2 \mathrm{~h})^{*}\end{array}$ & 61 \\
\hline $\mathrm{g}-\mathrm{C}_{3} \mathrm{~N}_{4}(<450)$ & Cr-doped $\mathrm{SrTiO}_{3}(<575)$ & 500 W Xe lamp $(>420)$ & & $\mathrm{De}(\mathrm{RhB})$ & $\eta_{\mathrm{RhB}}=99 \%(30 \mathrm{~min})^{*}$ & 62 \\
\hline g- $\mathrm{C}_{3} \mathrm{~N}_{4}(<460)$ & $\mathrm{Ag}_{2} \mathrm{CO}_{3}(<560)^{*}$ & 300 W Xe lamp (> 400) & $\mathrm{Ag}$ & $\mathrm{De}(\mathrm{RhB})$ & nearly $100 \%$ (40 min) & 63 \\
\hline $\mathrm{g}-\mathrm{C}_{3} \mathrm{~N}_{4}(<455)$ & N-doped $\mathrm{SrTiO}_{3}(<416)$ & $300 \mathrm{~W}$ Xe lamp (400-700) & & $\mathrm{De}(\mathrm{RhB})$ & $\eta_{\mathrm{RhB}}=98 \%(30 \mathrm{~min})^{*}$ & 64 \\
\hline $\mathrm{g}-\mathrm{C}_{3} \mathrm{~N}_{4}(<476)$ & $\mathrm{BiVO}_{4}(<506)$ & 500 W Xe lamp (> 420) & & $\mathrm{De}(\mathrm{RhB})$ & $\eta_{\mathrm{RhB}}=85 \%(5 \mathrm{~h})$ & 65 \\
\hline $\mathrm{g}-\mathrm{C}_{3} \mathrm{~N}_{4}(<446)$ & $\operatorname{AgBr}(<494)$ & 300 W Xe lamp $(>420)$ & & De $(\mathrm{MO})$ & $\eta_{\mathrm{MO}}=78.9 \%(2 \mathrm{~h})$ & 66 \\
\hline $\mathrm{g}-\mathrm{C}_{3} \mathrm{~N}_{4}(<460)$ & $\mathrm{MoO}_{3}(<442)$ & 350 W Xe lamp $(>420)$ & & $\mathrm{De}(\mathrm{MO})$ & $\eta_{\mathrm{MO}}=88 \%(2 \mathrm{~h})^{*}$ & 67 \\
\hline $\mathrm{g}-\mathrm{C}_{3} \mathrm{~N}_{4}(<461)$ & $\mathrm{BiOCl}(<344)$ & $300 \mathrm{~W}$ Xe lamp $(>420)$ & & $\mathrm{De}(\mathrm{RhB})$ & $\eta_{\mathrm{RhB}}=99 \%(1 \mathrm{~h})$ & 68 \\
\hline $\mathrm{g}-\mathrm{C}_{3} \mathrm{~N}_{4}(<460)$ & $\mathrm{Ag}_{3} \mathrm{PO}_{4}(<506)$ & 300 W Xe-arc lamp (420-800) & & De $(\mathrm{MO})$ & $\eta_{\mathrm{MO}}=96.8 \%(0.5 \mathrm{~h})$ & 69 \\
\hline $\mathrm{g}-\mathrm{C}_{3} \mathrm{~N}_{4}(<460)$ & sulfur-doped $\mathrm{TiO}_{2}(<450)$ & $500 \mathrm{~W}$ Xe lamp (> ca 400) & & $\mathrm{CO}_{2}$ reduction & $708 \times 10^{-6}(24 \mathrm{~h})$ & 70 \\
\hline $\mathrm{Bi}_{2} \mathrm{WO}_{6}(<451)$ & $\mathrm{g}-\mathrm{C}_{3} \mathrm{~N}_{4}(<461)$ & 300 W Xe lamp (> 420) & & $\mathrm{CO}_{2}$ reduction & CO yield $4.15 \mu \mathrm{mol}(8 \mathrm{~h})$ & 71 \\
\hline $\mathrm{g}-\mathrm{C}_{3} \mathrm{~N}_{4}(<460)$ & $\mathrm{SnO}_{2-\mathrm{x}}(<496)$ & 350 W Xe lamp (420-800) & & $\mathrm{CO}_{2}$ reduction & $22.7 \mu \mathrm{mol} \cdot \mathrm{h}^{-1} \cdot \mathrm{g}^{-1}$ & 72 \\
\hline $\mathrm{g}-\mathrm{C}_{3} \mathrm{~N}_{4}(<473)$ & $\mathrm{Ag}_{3} \mathrm{PO}_{4}(<523)$ & 500 W Xe lamp (> 420) & $\mathrm{Ag}$ & $\mathrm{CO}_{2}$ reduction & $57.5 \mu \mathrm{mol} \cdot \mathrm{h}^{-1} \cdot \mathrm{g}^{-1}$ & 73 \\
\hline $\begin{array}{l}\text { sulfur-doped } \\
\text { g- } \mathrm{C}_{3} \mathrm{~N}_{4}(<450)\end{array}$ & $\mathrm{CdS}(<560)$ & 300 W Xe lamp $(>420)$ & $\mathrm{Au}$ & water splitting & $\mathrm{H}_{2}$ yield $530 \mu \mathrm{mol}(5 \mathrm{~h})$ & 74 \\
\hline $\mathrm{g}-\mathrm{C}_{3} \mathrm{~N}_{4}(<462)$ & $\mathrm{Ag}_{3} \mathrm{PO}_{4}(<550)^{*}$ & LED visible-light & $\mathrm{Ag}$ & water splitting & $\begin{array}{c}\mathrm{O}_{2} \text { yield } \\
20 \mu \mathrm{mol} \cdot \mathrm{L}^{-1}(20 \mathrm{~min})\end{array}$ & 75 \\
\hline $\mathrm{g}-\mathrm{C}_{3} \mathrm{~N}_{4}(<460)$ & $\mathrm{WO}_{3}(<443)$ & $250 \mathrm{~W}$ UV-Vis lamp $(>420)$ & $\mathrm{rGO}^{\mathrm{h}}$ & water splitting & $\begin{array}{c}\mathrm{O}_{2} \text { yield ca } 17.5 \mu \mathrm{mol} \\
\mathrm{H}_{2} \text { yield } 35 \mu \mathrm{mol}(20 \mathrm{~h})\end{array}$ & 76 \\
\hline
\end{tabular}

The calculation formula for the available wavelength $(\lambda)$ in the table is $1240 / E_{\mathrm{g}}$, the data with $*$ is the estimated value of the curve in the graph. (a) De: degradation, (b) $\eta$ : degradation efficiency, (c) INC: Incandescent lamp, (d) TC: tetracycline, (e) RhB: rhodamine B,

(f) MO: methyl orange, (g) MB: methylene blue, (h) rGO: reduced graphene oxide

催化剂对甲醛有很好的分解效果。甲醛光分解过 程中, 羟基自由基 $(\cdot \mathrm{OH})$ 起主要的作用, $\mathrm{g}-\mathrm{C}_{3} \mathrm{~N}_{4}$ 的 价带电势过低不能将 $\mathrm{OH}^{-}$或 $\mathrm{H}_{2} \mathrm{O}$ 氧化为 $\cdot \mathrm{OH}^{\circ} \mathrm{TiO}_{2}$ 的电子空穴-复合率很高。实际参与光催化反应的 电子空穴仅占很小比例，对甲醛的光催化分解性 能也不好。两者复合(质量比 $1: 1$ )后构成 Z 型结 构, 电子和空穴在空间上分离开来，表现出了优 异的光分解甲醛性能。图 4(a)为复合材料光催化分 解甲醛的电荷传递示意图, $\mathrm{g}-\mathrm{C}_{3} \mathrm{~N}_{4}$ 导带处的电子与 空气中的氧气结合生成超氧自由基 $\left(\cdot \mathrm{O}^{2-}\right), \mathrm{TiO}_{2}$ 价 带的空穴与水反应生成· $\mathrm{OH}$, 甲醛在 $\mathrm{TiO}_{2}$ 的价带 与 $\cdot \mathrm{OH}$ 反应, 最终产物为 $\mathrm{CO}_{2}$ 和 $\mathrm{H}_{2} \mathrm{O}$ 。图 4(b) 为 $\mathrm{g}$ $\mathrm{C}_{3} \mathrm{~N}_{4} / \mathrm{TiO}_{2}$ 的结构示意图, 图 4(c)说明若 $\mathrm{g}-\mathrm{C}_{3} \mathrm{~N}_{4}$ 的含
量过高, 会破坏 $Z$ 型结构, 使得光催化性能降低。 光催化氧化甲醛的化学方程式如下:

$$
\mathrm{HCHO}+\mathrm{O}_{2} \stackrel{h v}{\rightarrow} \mathrm{CO}_{2}+\mathrm{H}_{2} \mathrm{O}
$$

\section{2 .2 降解有机物}

有机污染物造成了严重的环境问题，对人类 的健康饮水构成了很大的威胁 ${ }^{88}$, 光催化剂对大量 的有机污染物均有较好的降解效果, 有望解决水 污染带来的环境问题，实现产业化生产应用。 Chen 等 ${ }^{89}$ 用球磨热处理法制备出 $\mathrm{Z}$ 型 $\mathrm{WO}_{3} / \mathrm{g}-\mathrm{C}_{3} \mathrm{~N}_{4}$ 光 催化剂, 在最佳配比的 $\mathrm{WO}_{3} / \mathrm{g}-\mathrm{C}_{3} \mathrm{~N}_{4}$ 可见光下 $2 \mathrm{~h}$ 对 $\mathrm{BF}$ 和 $\mathrm{MB}$ 的分解率高达 $94.1 \%$ 和 $98.5 \%$ ，而同等条 件下 $\mathrm{g}-\mathrm{C}_{3} \mathrm{~N}_{4}$ 对 $\mathrm{BF}$ 和 $\mathrm{MB}$ 的分解率均只有 $50 \%$ 左 右。Tian 等 ${ }^{65}$ 采用混合煅烧法制备出了 $\mathrm{Z}$ 型 $\mathrm{BiVO}_{4} /$ 


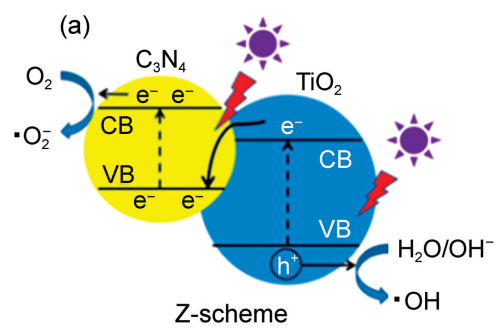

(b)
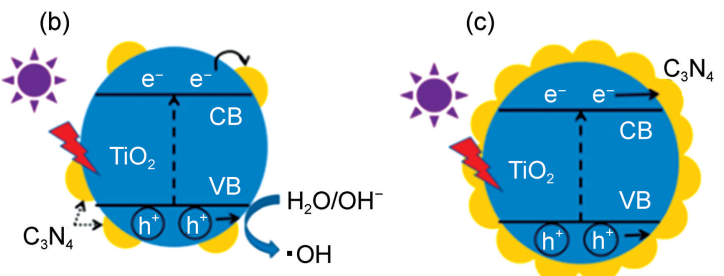

图 4 紫外光照下 $\mathrm{Z}$ 型 $\mathrm{g}-\mathrm{C}_{3} \mathrm{~N}_{4}-\mathrm{TiO}_{2}$ 光催化剂的 电荷传递与分离示意图 ${ }^{55}$

Fig.4 Schematic illustration for the charge transfer and separation in $\mathrm{g}_{-} \mathrm{C}_{3} \mathrm{~N}_{4}-\mathrm{TiO}_{2} \mathrm{Z}$-scheme photocatalysts under UV light irradiation ${ }^{55}$

$\mathrm{g}-\mathrm{C}_{3} \mathrm{~N}_{4}$ 光催化剂。最佳配比的 $\mathrm{BiVO}_{4} / \mathrm{g}-\mathrm{C}_{3} \mathrm{~N}_{4}$ 降解罗 丹明 $\mathrm{B}(\mathrm{RhB})$ 的表观速率常数 $(k)$ 为 $0.342 \mathrm{~h}^{-1}$, 约为 纯 $\mathrm{BiVO}_{4}$ 或 $\mathrm{g}-\mathrm{C}_{3} \mathrm{~N}_{4}$ 的 10 倍。 $\mathrm{Z}-\mathrm{g}-\mathrm{C}_{3} \mathrm{~N}_{4}$ 光催化剂降解 有机物的反应原理可以归纳表述为如下方程。

PS II 价带上的反应:

$$
\mathrm{h}^{+}+\text {organic pollutants } \stackrel{h v}{\rightarrow} \text { products }
$$
或

$$
\begin{aligned}
& \mathrm{H}_{2} \mathrm{O}+\mathrm{h}^{+} \stackrel{h v}{\rightarrow} \cdot \mathrm{OH} \\
& \cdot \mathrm{OH}+\text { organic pollutants } \stackrel{h v}{\rightarrow} \text { products }
\end{aligned}
$$

PS I 导带上的反应:

$$
\begin{aligned}
& \mathrm{O}_{2}+\mathrm{e}^{-} \stackrel{h v}{\rightarrow} \mathrm{O}_{2}^{--} \\
& \mathrm{O}_{2}^{--}+\text {organic pollutants } \stackrel{h v}{\rightarrow} \text { products }
\end{aligned}
$$

在某些 Z-g- $\mathrm{C}_{3} \mathrm{~N}_{4}$ 光催化体系中, PS II 组分的 金属元素会在光催化的过程中被还原为金属单 质, 镶嵌在 PS I 与 PS II 之间, 形成导电介质减小 电阻, 提高光催化性能 ${ }^{64}$ 。此类常见的 PS II 组分有 $\mathrm{Ag}$ 化合物, 如 $\mathrm{Ag}_{2} \mathrm{CO}_{3} 、 \mathrm{Ag}_{3} \mathrm{PO}_{4} 、 \mathrm{AgBr}$ 等。 $\mathrm{Shi}$ 等 ${ }^{63}$ 合成了 $\mathrm{Z}$ 型 $\mathrm{Ag}_{2} \mathrm{CO}_{3} / g-\mathrm{C}_{3} \mathrm{~N}_{4}$ 光催化材料, 如图 5 所示, 在光催化的过程中 $\mathrm{Ag}_{2} \mathrm{CO}_{3}$ 分解产生了金属 $\mathrm{Ag}, \mathrm{Ag}$ 在 $\mathrm{Ag}_{2} \mathrm{CO}_{3}$ 和 $\mathrm{g}_{-} \mathrm{C}_{3} \mathrm{~N}_{4}$ 之间传输电荷, 复合物 在可见光区域的光吸收增强。在 $40 \mathrm{~min}$ 可见光照 射下，对 RhB 的分解率接近 $100 \%$ 。

\section{3 还原 $\mathrm{CO}_{2}$}

$\mathrm{CO}_{2}$ 是温室气体的主要成分, 利用光催化技术 还原 $\mathrm{CO}_{2}$ 不仅可以将其转化为燃料, 而且可以很好 $\ominus$ : electrons

$\oplus$ : holes

$\bullet:$ RhB

: degradation products

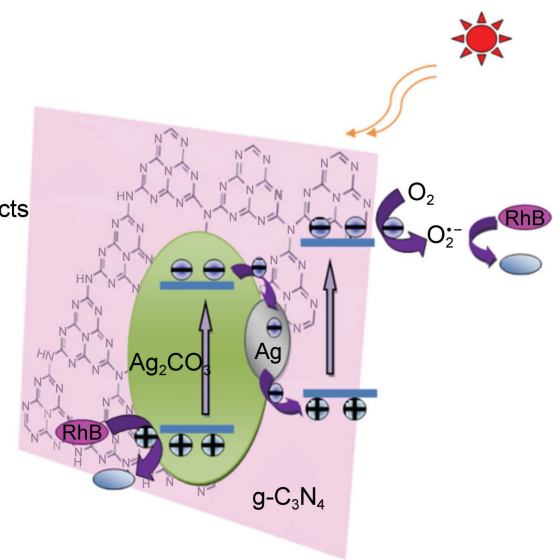

图 $5 \mathrm{~g}-\mathrm{C}_{3} \mathrm{~N}_{4} / \mathrm{Ag}_{2} \mathrm{CO}_{3}$ 复合物光催化分解 $\mathrm{RhB}^{63}$

Fig.5 Proposed photodegradation RhB on g- $\mathrm{C}_{3} \mathrm{~N}_{4} / \mathrm{Ag}_{2} \mathrm{CO}_{3}$ composite $^{63}$

地解决全球变暖问题 ${ }^{90,91}$ 。但 $\mathrm{CO}_{2}$ 的能级较低、异 常稳定, 很难将其直接作为反应原料用于工业生 产 ${ }^{22}$ 。科学工作者们在半导体还原 $\mathrm{CO}_{2}$ 方面做了大 量的研究工作 ${ }^{70-73}$ 。 $\mathrm{He}$ 等 $^{72}$ 运用煅烧法制备出具有 高光催化活性的 $\mathrm{Z}$ 型 $\mathrm{SnO}_{2-x} / \mathrm{g}-\mathrm{C}_{3} \mathrm{~N}_{4}\left(\mathrm{SnO}_{2-x}\right.$ 质量分数 为 $42.2 \%$ ) 光催化材料, 同等条件下 $\mathrm{SnO}_{2-x} / \mathrm{g}-\mathrm{C}_{3} \mathrm{~N}_{4}$ 光 催化还原 $\mathrm{CO}_{2}$ 的能力是 $\mathrm{P} 25$ 的 5 倍。如图 6 所示, $\mathrm{CO}_{2}$ 转化为 $\mathrm{CH}_{3} \mathrm{OH} 、 \mathrm{CH}_{4}$ 和 $\mathrm{CO}$, 显然 $\mathrm{CO}_{2} /$ $\mathrm{CH}_{3} \mathrm{OH} 、 \mathrm{CO}_{2} / \mathrm{CO}$ 等的还原电势比光解水的电势还 要负, 其反应对半导体的要求更高, 反应更难进 行。该体系中 $\mathrm{CO}_{2}$ 转化的光化学反应可以表述为如 下方程:

$$
\mathrm{CO}_{2}+6 \mathrm{H}^{+}+6 \mathrm{e}^{-} \stackrel{h v}{\rightarrow} \mathrm{CH}_{3} \mathrm{OH}+\mathrm{H}_{2} \mathrm{O}
$$

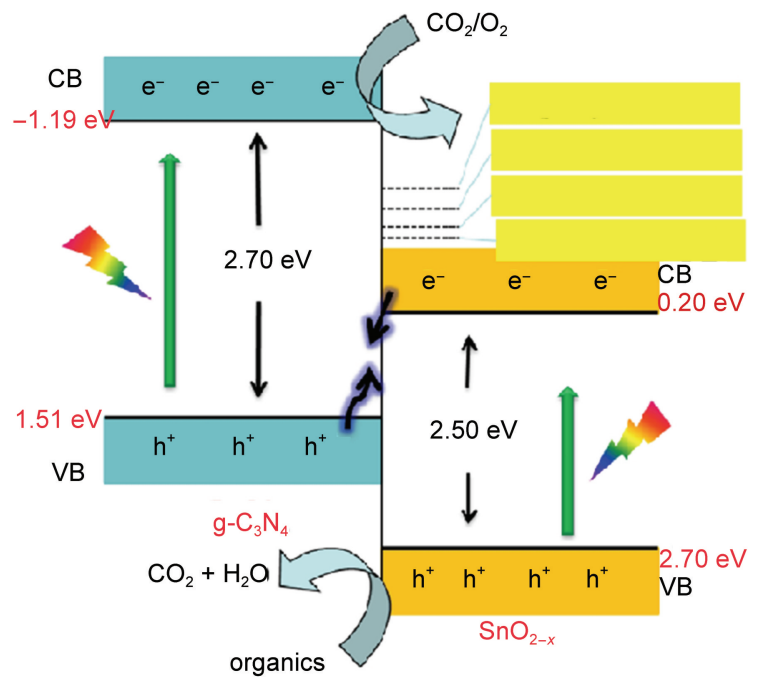

图 6 可见光下 $\mathrm{SnO}_{2-x} / \mathrm{g}_{-} \mathrm{C}_{3} \mathrm{~N}_{4}$ 电荷传递机理 ${ }^{73}$

Fig.6 Possible electron tranfer mechanism for $\mathrm{SnO}_{2-x} / \mathbf{g}-\mathrm{C}_{3} \mathrm{~N}_{4}$ under visible light illumination ${ }^{73}$ 


$$
\begin{aligned}
& \mathrm{CO}_{2}+2 \mathrm{H}^{+}+2 \mathrm{e}^{-} \stackrel{h v}{\rightarrow} \mathrm{CO}+\mathrm{H}_{2} \mathrm{O} \\
& \mathrm{CO}_{2}+8 \mathrm{H}^{+}+8 \mathrm{e}^{-} \stackrel{h v}{\rightarrow} \mathrm{CH}_{4}+2 \mathrm{H}_{2} \mathrm{O} \\
& \mathrm{H}_{2} \mathrm{O}+2 \mathrm{~h}^{+} \stackrel{h v}{\rightarrow} 2 \mathrm{H}^{+}+\frac{1}{2} \mathrm{O}_{2}
\end{aligned}
$$

由方程(12)-(15)可知, $\mathrm{SnO}_{2-x} / \mathrm{g}-\mathrm{C}_{3} \mathrm{~N}_{4}$ 光催化还 原 $\mathrm{CO}_{2}$ 的过程中, 光生电子与光生空穴在反应中起 决定性的作用。

$\mathrm{Yu}$ 等 ${ }^{58}$ 以尿素和六水合硝酸锌为原料, 通过简 单的混合、干燥、焙烧等操作在合成了 $\mathrm{Z}$ 型 $\mathrm{g}$ $\mathrm{C}_{3} \mathrm{~N}_{4} / \mathrm{ZnO}$ 复合材料, $\mathrm{ZnO}$ 组分镶嵌在 $\mathrm{g}-\mathrm{C}_{3} \mathrm{~N}_{4}$ 的介 孔以及层间, 使得复合物的结构十分紧密, 提高 了电荷在组分间的分离、传输效率。 $\mathrm{ZnO}$ 与 $\mathrm{g}_{-} \mathrm{C}_{3} \mathrm{~N}_{4}$ 之间的耦合作用使得复合物的光吸收较纯 $g-\mathrm{C}_{3} \mathrm{~N}_{4}$ 而 言在紫外和可见光区域都有较大的增强。g- $\mathrm{C}_{3} \mathrm{~N}_{4} /$ $\mathrm{ZnO}$ 复合材料在紫外光下还原 $\mathrm{CO}_{2}$ 的速率为纯 $\mathrm{ZnO}$ 或 $\mathrm{g}-\mathrm{C}_{3} \mathrm{~N}_{4}$ 的两倍多。目前很多文献报道了构建 Z-g$\mathrm{C}_{3} \mathrm{~N}_{4}$ 光催化体系提高 $\mathrm{CO}_{2}$ 的转化率, 如 $\mathrm{g}-\mathrm{C}_{3} \mathrm{~N}_{4} /$ $\mathrm{Bi}_{2} \mathrm{WO}_{6}{ }^{71}$ 和 $\mathrm{g}-\mathrm{C}_{3} \mathrm{~N}_{4} / \mathrm{WO}_{3}{ }^{93}$ 等, 但以 $\mathrm{CO}_{2}$ 为原料进行 工业生产依然难以实现, 任重道远。

\section{4 影响 Z-g- $\mathrm{C}_{3} \mathrm{~N}_{4}$ 光催化性能的因素}

\section{$4.1 \mathrm{pH}$ 值}

在光催化反应过程中, $\mathrm{pH}$ 值对光催化反应的 活性有很大影响。大多数光催化反应体系在中性 条件下进行, 部分体系在酸性条件下表现出更好 的光催化性能 ${ }^{94}$, 部分体系则在碱性条件下更有 利 ${ }^{95}$ 。 $\mathrm{pH}$ 值对光催化反应体系的影响可以分为三 类: (a) 不同 $\mathrm{pH}$ 值条件下半导体光催化剂的导带、 价带位置会发生变化, 禁带宽度也会有所差异 ${ }^{96}$; (b) $\mathrm{pH}$ 值不同时, 半导体表面电荷的正负性会发生 变化, 影响光催化剂对降解物的吸附能力 ${ }^{97}$; (c) $\mathrm{pH}$ 值不同时, 在动力学上 $\mathrm{H}^{+}$或 $\mathrm{OH}^{-}$会促进或抑制 体系中光催化活性因子 $\left(\right.$ 如 $\left.\cdot \mathrm{OH}, \cdot \mathrm{O}^{2-}\right)$ 的生成。 $\mathrm{He}$ 等 ${ }^{67}$ 报道了碱性条件下 $\mathrm{MoO}_{3} / \mathrm{g}-\mathrm{C}_{3} \mathrm{~N}_{4}$ 的光催化性能 更差, 该研究表明 $\mathrm{MoO}_{3} / \mathrm{g}-\mathrm{C}_{3} \mathrm{~N}_{4}$ 在酸性或者中性条 件下对 MO 的光降解性能更好 ${ }^{70}$ 。

Zhao 等 ${ }^{76}$ 研究了不同 $\mathrm{pH}$ 值条件下 $\mathrm{C}_{3} \mathrm{~N}_{4} / \mathrm{rGO} /$ $\mathrm{WO}_{3}$ 体系的光催化产氢量。实验结果表明在一定范 围内, $\mathrm{pH}$ 值越高, 氢气产率越高。他们推断在水分 解的过程中, 虽然酸性条件有利于产氢, 但却不利 于氧气在 $\mathrm{WO}_{3}$ 的表面生成, 而在该水解体系中产氧 反应占了主导地位, 故而碱性条件更有利。

\section{2 导电介质}

导电介质的主要作用是在 PS I 和 PS II 之间传 输电荷 ${ }^{98,99}$, 常见的导电介质为金属单质, 如 $\mathrm{Ag}$ 、 $\mathrm{Au} 、 \mathrm{Cd}$ 等 ${ }^{100,101}$ 。 $\mathrm{Li}$ 等 ${ }^{74}$ 制备了 $\mathrm{Z}$ 型 $\mathrm{g}-\mathrm{CNS} / \mathrm{Au} / \mathrm{CdS}$ 光催化剂, $\mathrm{Au}$ 在 $\mathrm{g}-\mathrm{CNS}$ 的价带与 $\mathrm{CdS}$ 的导带之间 传递电荷, 光电子寿命延长。 $\mathrm{g}-\mathrm{CNS} / \mathrm{Au} / \mathrm{CdS}$ 光降 解 $\mathrm{RhB}$, 产氢性能均优于 $\mathrm{g}-\mathrm{CNS} / \mathrm{CdS}$ 。导电介质对 $\mathrm{Z}$ 型体系光催化性能的影响与导电介质的含量密切 相关, 当导电介质的含量超过一定范围时, 反而 抑制光催化性能, 可能与金属单质占据了 PS I 和 PS II 上面的活性位置有关 ${ }^{102}$ 。

在部分 Z-g- $\mathrm{C}_{3} \mathrm{~N}_{4}$ 体系中, 导电介质与 PS II 组 分有相同的金属元素, 按照导电介质的成因可划 分为两种类型。一类体系中导电介质是通过物理 化学手段复合进入体系, Yang 等 ${ }^{60}$ 采用化学沉积法 制备出了 $\mathrm{Ag} @ \mathrm{AgBr} / \mathrm{g}-\mathrm{C}_{3} \mathrm{~N}_{4}$ 光催化剂, 该体系在可 见光下表现出强还原性; 另一类体系中导电介质 是由于 PS II 不稳定分解产生, Katsumata 等 ${ }^{103}$ 采用 原位还原沉淀法制备了 $\mathrm{g}-\mathrm{C}_{3} \mathrm{~N}_{4} / \mathrm{Ag}_{3} \mathrm{PO}_{4}$ 光催化剂, 在光催化的过程中有 $\mathrm{Ag}$ 生成, 镶嵌在 $g-\mathrm{C}_{3} \mathrm{~N}_{4}$ 和 $\mathrm{Ag}_{3} \mathrm{PO}_{4}$ 的界面处。最佳比例的 $\mathrm{g}-\mathrm{C}_{3} \mathrm{~N}_{4} / \mathrm{Ag}_{3} \mathrm{PO}_{4}$ 复合 物在可见光照射下, $5 \mathrm{~min}$ 就可将 $\mathrm{MO}$ 完全降解。

非金属导体也可以充当 Z-g- $\mathrm{C}_{3} \mathrm{~N}_{4}$ 的导电介质, 在合适的体系中对光催化性能会有很大的提升。 Zhao 等 ${ }^{76}$ 设计合成了 $\mathrm{C}_{3} \mathrm{~N}_{4}-\mathrm{rGO}-\mathrm{WO}_{3}$ 光催化剂, 该 体系中有 $1 \%(w)$ 的 Pt沉积在复合物表面, 在光催化 反应中 $\mathrm{Pt}$ 仅仅起助催化剂的作用, 石墨烯是真正 意义上的导电介质。如图 7 所示, $\mathrm{C}_{3} \mathrm{~N}_{4}-\mathrm{WO}_{3}$ 体系中

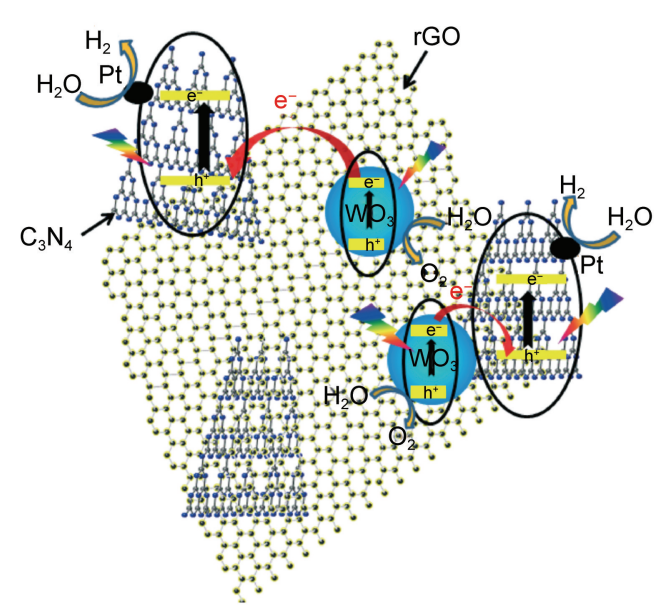

图 $7 \quad \mathrm{C}_{3} \mathrm{~N}_{4}-\mathrm{WO}_{3}$ 复合物在有无还原石墨烯条件下 光催化分解水的机理图 ${ }^{76}$

Fig.7 Proposed mechanism for the photocatalytic decomposition of water by $\mathrm{C}_{3} \mathrm{~N}_{4}-\mathrm{WO}_{3}$ composite with or without the mediation of rGO$^{76}$ 

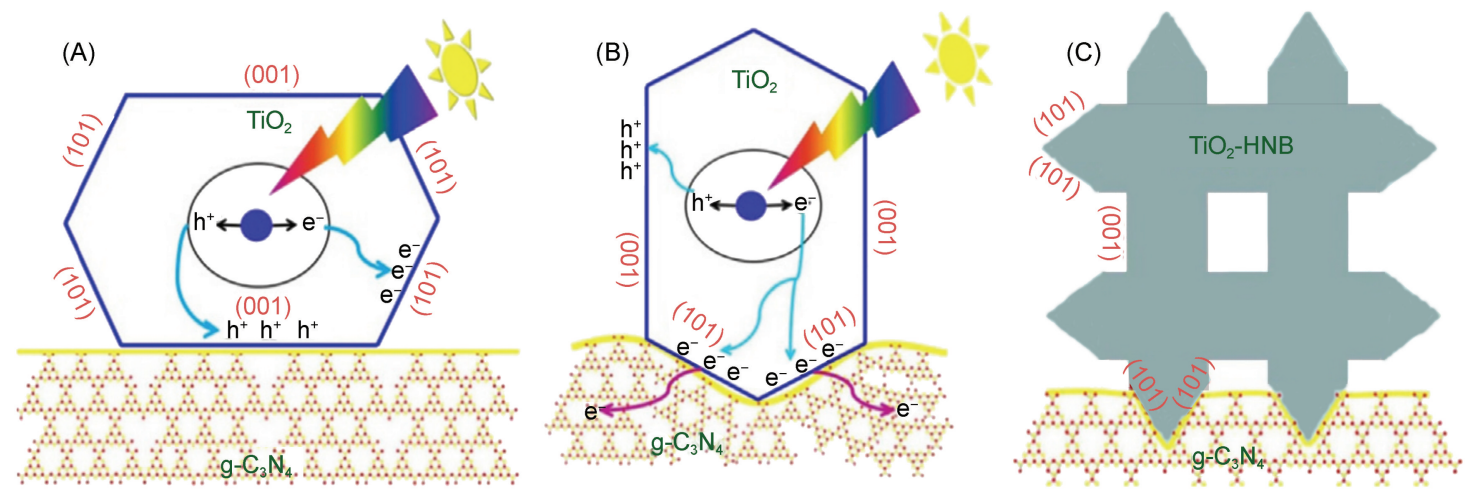

图 $8 \mathrm{TiO}_{2}$ 中空纳米盒不同晶面与 $\mathrm{g}-\mathrm{C}_{3} \mathbf{N}_{4}$ 接触的光催化过程 ${ }^{105}$

Fig.8 Photo-generated carriers distribution schematic diagram at contact interface of $g-C_{3} \mathrm{~N}_{4}$ and different facets of $\mathrm{TiO}_{2}$ hollow nanoboxs ${ }^{105}$

电子直接由 $\mathrm{WO}_{3}$ 的导带传递至 $\mathrm{C}_{3} \mathrm{~N}_{4}$ 的价带, 当体 系中有石墨烯时, 电子会从 $\mathrm{WO}_{3}$ 的导带转移至石墨 烯, 然后再转移到 $\mathrm{C}_{3} \mathrm{~N}_{4}$ 的价带, 抑制了 $\mathrm{C}_{3} \mathrm{~N}_{4}$ 和 $\mathrm{WO}_{3}$ 自身的电子空穴复合。中性条件下, $\mathrm{C}_{3} \mathrm{~N}_{4}-\mathrm{rGO}-\mathrm{WO}_{3}$ 体系的光催化产氢性能远优于 $\mathrm{C}_{3} \mathrm{~N}_{4}-\mathrm{WO}_{3}$ 体系。与 金属导电介质相似, 石墨烯的量必须控制在合适 的范围区间内。

\section{3 结 构}

光催化材料的性能与结构密不可分, 同一材 料因结构不同表现出的性能差异很大 ${ }^{104}$ 。改变光催 化材料的结构很可能会改变光催化反应的机理, 从而使得性能差异大。Chen 等 ${ }^{57}$ 合成了 $\mathrm{Z}$ 型 $\mathrm{g}$ $\mathrm{C}_{3} \mathrm{~N}_{4}-\mathrm{Ag}_{3} \mathrm{PO}_{4}$ 光催化剂, 当某一组分含量过多时, 会使得光催化反应机理发生变化。其结构类似于 图 4(b)与图 4(c), $\mathrm{g}-\mathrm{C}_{3} \mathrm{~N}_{4}$ 过量时会包覆在 $\mathrm{Ag}_{3} \mathrm{PO}_{4}$ 的 表面, $\mathrm{Ag}_{3} \mathrm{PO}_{4}$ 不能被光激发故无法产生电子跃 迁, 不能构成 Z 型结构。

类似于上述结构的影响, 材料的微观结构同 样对性能有很大的影响。PS II 组分不同的晶面与 $\mathrm{g}-\mathrm{C}_{3} \mathrm{~N}_{4}$ 接触也使得性能差异很大, Huang 等 ${ }^{105}$ 采用 微波辅助法制备出了 $\mathrm{Z}$ 型 $\mathrm{g}-\mathrm{C}_{3} \mathrm{~N}_{4} / \mathrm{TiO}_{2}\left(\mathrm{TiO}_{2}\right.$ 为空心 纳米盒子结构)光催化剂, $\mathrm{TiO}_{2}$ 受光激发, 空穴转 移至(001)晶面而电子则激发到(101)晶面。若材料 结构如图 8(A) 所示, $\mathrm{TiO}_{2}$ 的 (001) 晶面与 $\mathrm{g}-\mathrm{C}_{3} \mathrm{~N}_{4}$ 接 触, 由于 $\mathrm{TiO}_{2}$ 的价带很低, 空穴无法跃迁到能量 相对较高的 $\mathrm{g}-\mathrm{C}_{3} \mathrm{~N}_{4}$ 价带处, 故而使得光催化性能不 佳。图 8(B)中, $\mathrm{TiO}_{2}$ 的导带电子处于激发态, 可以 转移至能量低的 $\mathrm{g}-\mathrm{C}_{3} \mathrm{~N}_{4}$ 价带处, 使得材料有强氧化 还原性。图 8(C) 基于图 8(B)的原理, 构建了空心纳 米盒子结构, 使得 $\mathrm{g}-\mathrm{C}_{3} \mathrm{~N}_{4}$ 仅与 $\mathrm{TiO}_{2}$ 的(101) 晶面接
触, 在光降解染料方面表现出了良好的性能。

4.4 缺陷

在 Z-g- $\mathrm{C}_{3} \mathrm{~N}_{4}$ 体系中, 目前已经报道的非本征缺 陷主要有元素掺杂和原子缺失两类。缺陷主要有 如下两点作用: (a) 形成缺陷能级 ${ }^{106}$, 在价带与导 带之间形成一个缺陷能级, 为电子跃迁提供一个 “跳板”; (b) 改变带隙宽度 ${ }^{107}$, 使得价带与导带的 位置更近, 便于电子在更低的光照能量下跃迁。 对于 $\mathrm{Z}-\mathrm{g}-\mathrm{C}_{3} \mathrm{~N}_{4}$ 体系, 适当的表面缺陷对其性能也有 一定的改善。 $\mathrm{Yin}^{108}$ 和 $\mathrm{Zang}{ }^{109}$ 等研究了 $\mathrm{g}^{-} \mathrm{C}_{3} \mathrm{~N}_{4} / \mathrm{SnO}_{2}$ 光催化体系, 实验数据分析其为异质结结构, 而 $\mathrm{He}$ 等 ${ }^{73}$ 制备出了部分氧原子晶格位置缺失型 $\mathrm{SnO}_{2-x}$, 其带隙结构与 $\mathrm{SnO}_{2}$ 不同, 与 $\mathrm{g}-\mathrm{C}_{3} \mathrm{~N}_{4}$ 复合后 构成了 $\mathrm{Z}$ 型光催化剂。Wang 等 ${ }^{110}$ 用 $\mathrm{S}$ 掺杂 $\mathrm{g}-\mathrm{C}_{3} \mathrm{~N}_{4}$ 后 其对可见光的吸收变强。Kumar 等 ${ }^{64}$ 用 $\mathrm{N}$ 掺杂改性 $\mathrm{SrTiO}_{3}$, 改变了其带隙的位置, 与 $\mathrm{g}-\mathrm{C}_{3} \mathrm{~N}_{4}$ 复合后构 成了 $\mathrm{Z}$ 型体系, 增强了可见光下的光催化性能。

\section{5 总结和展望}

$\mathrm{Z}$ 型光催化体系在光解水、还原 $\mathrm{CO}_{2}$ 、分解有 机物等方面均有良好的性能, g- $\mathrm{C}_{3} \mathrm{~N}_{4}$ 是近年来研究 的热门光催化材料。基于 $\mathrm{g}-\mathrm{C}_{3} \mathrm{~N}_{4}$ 的 $\mathrm{Z}$ 型光催化体系 表现出了优异的光催化性能, 有着巨大的应用潜 力。近年来科学工作者们对 Z-g- $\mathrm{C}_{3} \mathrm{~N}_{4}$ 体系的研究取 得了很大进步, 但仍有诸多地方难以解释清楚, 体系机理研究仍有不足, 存在如下几个问题仍需 解决:

(1) 抑制逆反应。在光分解水体系中, PS I 上 产氢 PS II 上产氧, 不同微粒在溶液中相互碰撞, 会使得不同微粒的产氢与产氧接触, 增大了逆反 
应的可能性。即使对于同一微粒, 由于尺寸仅在 微米、纳米级别, 产生的氢气与氧气也很容易接 触, 逆反应也很难避免。笔者构想, 采用物理化 学方法, 将 PS I 和 PS II 光催化剂分别固定在导电 薄膜的两侧, 构成 Z 型结构, 产氢与产氧在两个独 立的区域发生, 可以避免氢气与氧气的结合。

(2) 提高回收率。 Z-g- $\mathrm{C}_{3} \mathrm{~N}_{4}$ 光催化剂若要实现 工业化生产，必须解决其回收的问题。目前使用 的 Z-g- $\mathrm{C}_{3} \mathrm{~N}_{4}$ 光催化剂主要以粉末的形式存在, 分散 在溶液中, 光催化反应以后回收困难。关于催化 剂回收的问题, 笔者认为可以从磁分离的角度着 手, 将磁性材料与 $\mathrm{g}-\mathrm{C}_{3} \mathrm{~N}_{4}$ 复合, 构成具有磁性的 $\mathrm{Z}-\mathrm{g}-\mathrm{C}_{3} \mathrm{~N}_{4}$ 光催化材料, 光催化反应完成后在磁场的 作用下分离, 快捷简单, 无二次污染。

(3) 提升循环稳定性。虽然大多数 Z $-g-\mathrm{C}_{3} \mathrm{~N}_{4}$ 光 催化剂较 $\mathrm{g}-\mathrm{C}_{3} \mathrm{~N}_{4}$ 循环稳定性有较大的提升, 但是多 次循环使用时其性能还是略有下降, 若要实现工 业应用，其循环稳定性能还有待大幅度提高。

(4) 扩大可见光吸收范围。大多数光催化剂都 只能利用极少的一部分太阳光, 严重限制了光催 化剂的产业化发展。扩大可见光吸收范围需缩小 PS I 与 PS II 的禁带宽度, 目前研究较多的是掺杂 改性, 但掺杂很难控制带隙的宽度, 而且性能也 无法保证，难以实现大规模生产制造。如何探寻 一种简单有效的改性方法, 有待深入研究。

\section{References}

(1) Xi, Z. H.; Li, C. J.; Zhang, L.; Xing, M. Y.; Zhang J. L. Int. J. Hydrog. Energy 2014, 39, 6345. doi: 10.1016/j. ijhydene.2014.01.209

(2) Ma, B. J.; Yang, J. H.; Han, H. X.; Wang, J. T.; Zhang, X. H.; Li, C. J. Phys. Chem. C 2010, 114, 12818. doi: 10.1021/ jp103722j

(3) Liu, J. H. Mod. Chem. Ind. 2006, 26 (2), 10. [刘江华. 现代化 工, 2006, 26 (2), 10.]

(4) Ladomenou, K.; Natali, M.; Iengo, E.; Charalampidis, G.; Scandola, F.; Coutsolelos, A. G. Coord. Chem. Rev. 2015, 304, 38.

(5) Zhang, N.; Zhang, Y. H.; Xu, Y. J. Nanoscale 2012, 4, 5792. doi: $10.1039 / \mathrm{c} 2 \mathrm{nr} 31480 \mathrm{k}$

(6) Wang, H. L.; Zhang, L. S.; Chen, Z. G.; Hu, J. Q.; Li, S. J.; Wang, Z. H.; Liu, J. S.; Wang, X. C. Chem. Soc. Rev. 2014, 43, 5234. doi: 10.1039/C4CS00126E

(7) Suarez, C. M.; Hernández, S.; Russo, N. Appl. Catal. A-Gen. 2015, 504, 158. doi: 10.1016/j.apcata.2014.11.044

(8) Das, S.; Daud, W. M. A. W. Renew. Sust. Energ. Rev. 2014, 39, 765. doi: 10.1016/j.rser.2014.07.046
(9) Grewe, T.; Tüysüz, H. ACS Appl. Mater. Interfaces 2015, 7, 23153. doi: 10.1021/acsami.5b06965

(10) Zhou, W.; Li, W.; Wang, J. Q.; Qu, Y.; Yang, Y.; Xie, Y.; Zhang, K. F.; Wang, L.; Fu, H. G.; Zhao, D. Y. J. Am. Chem. Soc. 2014, 136, 9280. doi: 10.1021/ja504802q

(11) Liu, B.; Liu, L. M.; Lang, X. F.; Wang, H. Y.; Lou, X. W.; Aydil, E. S. Energy Environ. Sci. 2014, 7, 2592. doi: 10.1039/ C4EE00472H

(12) Tu, W. G.; Zhou, Y.; Zou, Z. G. Adv. Mater. 2014, 26, 4607. doi: 10.1002/adma.v26.27

(13) Jiang, R. B.; Li, B. X.; Fang, C. H.; Wang, J. F. Adv. Mater. 2014, 26, 5274. doi: 10.1002/adma.201400203

(14) Yu, J. G.; Low, J. X.; Xiao, W.; Zhou, P.; Jaroniec, M. J. Am. Chem. Soc. 2014, 136, 8839. doi: 10.1021/ja5044787

(15) Sun, J. J.; Li, X. Y.; Zhao, Q. D.; Ke, J.; Zhang, D. K. J. Phys. Chem. C 2014, 118, 10113. doi: 10.1021/jp5013076

(16) Quici, N.; Vera, M. L.; Choi, H.; Puma, G. L.; Dionyisou, D. D.; Litter, M. I.; Destaillats, H. Appl. Catal. B 2010, 95, 312. doi: 10.1016/j.apcatb.2010.01.009

(17) Nie, L. H.; Yu, J. G.; Li, X. Y.; Cheng, B.; Liu, G.; Jaroniec, M. Environ. Sci. Technol. 2013, 47, 2777. doi: 10.1021/es3045949

(18) Zhang, G.; Kim, G.; Choi, W. Energy Environ. Sci. 2014, 7, 954. doi: 10.1039/c3ee43147a

(19) Wang, C. C.; Li, J. R.; Lv, X. L.; Zhang, Y. Q.; Guo, G. S. Energy Environ. Sci. 2014, 7, 2831. doi: 10.1039/C4EE01299B

(20) Soares, P. A.; Silva, T. F. C. V.; Manenti, D. R.; Souza, S. M. A. G. U.; Boaventura, R. A. R.; Vilar, V. J. P. Environ. Sci. Pollut. Res. 2014, 21, 932. doi: 10.1007/s11356-013-1934-0

(21) Lei, Y. B.; Liu, P. C.; Zhu, W. W.; Zhang, J. Y.; Du, D. L.; Xiao, X. Environ. Prot. Chem. Ind. 2015, 35 (3), 253. [ [雷育斌, 刘鹏程, 朱雯雯, 章佳荣, 杜道林, 肖 翔. 化工环保, 2015, 35 (3), 253.]

(22) Fujishima, A.; Honda, K. Nature 1972, 238, 37. doi: 10.1038/ $238037 \mathrm{a} 0$

(23) Liu, D.; Li, Z. H.; Wang, W. Q.; Wang, G. Q.; Liu, D. J. Alloy. Compd. 2016, 654, 491. doi: 10.1016/j.jallcom.2015.09.140

(24) Niu, P.; Liu, G.; Cheng, H. M. J. Phys. Chem. C 2012, 116, 11013. doi: $10.1021 / \mathrm{jp} 301026 \mathrm{y}$

(25) Zhang, J. S.; Wang, B.; Wang, X. C. Acta Phys. -Chim. Sin. 2013, 29 (9), 1865. [张金水, 王 博, 王心晨. 物理化学学报, 2013, 29 (9), 1865.] doi: 10.3866/PKU.WHXB201306173

(26) Cao, S. W.; Low, J. X.; Yu, J. G.; Jaroniec, M. Adv. Mater. 2015, 27, 2150. doi: 10.1002/adma.201500033

(27) Zheng, Y.; Lin, L. H.; Wang, B.; Wang, X. C. Angew. Chem. Int. Edit. 2015, 54, 12868. doi: 10.1002/anie.v54.44

(28) Wang, X. C.; Maeda, K.; Thomas, A.; Takanabe, K.; Xin, G.; Carlsson, J. M.; Domen, K.; Antonietti, M. Nature Mater. 2009, 8, 76. doi: 10.1038/nmat2317

(29) Yan, S. C.; Lv, S. B.; Li, Z. S.; Zou, Z. G. Dalton Trans. 2010, 39, 1488. doi: 10.1039/B914110C

(30) Dong, F.; Wu, L. W.; Sun, Y. J.; Fu, M.; Wu, Z. B.; Lee, S. C. 
J. Mater. Chem. 2011, 21, 15171. doi: 10.1039/c1jm12844b

(31) Ma, L. T.; Fan, H. Q.; Li, M. M.; Tian, H.; Fang, J. W.; Dong, G. Z. J. Mater. Chem. A 2015, 3, 22404. doi: 10.1039/ C5TA05850C

(32) Wang, Y.; Zhang, J. S.; Wang, X. C.; Antonietti, M.; Li, H. Angew. Chem. Int. Edit. 2010, 49, 3356. doi: 10.1002/ anie. 201000120

(33) Zhang, Y. J.; Mori, T.; Niu, L.; Ye, J. H. Energy Environ. Sci. 2011, 4, 4517. doi: 10.1039/clee01400e

(34) Liu, G.; Niu, P.; Sun, C. H.; Smith, S. C.; Chen, Z. G.; Lu, G. Q.; Cheng, H. M. J. Am. Chem. Soc. 2010, 132, 11642. doi: $10.1021 / \mathrm{ja} 103798 \mathrm{k}$

(35) Zhou, P.; Wu, J. H.; Yu, W. L.; Zhao, G. H.; Fang, G. J.; Cao, S. W. Appl. Surf. Sci. 2014, 319, 167. doi: 10.1016/j. apsusc.2014.05.045

(36) Lo, C. C.; Huang, C. W.; Liao, C. H.; Wu, J. C. S. Int. J. Hydrog. Energy 2010, 35, 1523. doi: 10.1016/j. ijhydene.2009.12.032

(37) Iwase, A.; Ng, Y. H.; Ishiguro, Y.; Kudo, A.; Amal, R. J. Am. Chem. Soc. 2011, 133, 11054. doi: 10.1021/ja203296z

(38) Chen, S. F.; Hu, Y. F.; Meng, S. G.; Fu, X. L. Appl. Catal. B 2014, 150, 564. doi: 10.1016/j.apcatb.2013.12.053

(39) Jin, Z. Y.; Murakami, N.; Tsubota, T.; Ohno, T. Appl. Catal. B 2014, 150, 479. doi: 10.1016/j.apcatb.2013.12.048

(40) Yun, H. J.; Lee, H.; Kim, N. D.; Lee, D. M.; Yu, S. J.; Yi, J. H. ACS Nano 2011, 5, 4084. doi: 10.1021/nn2006738

(41) Wu, W. H. Phytophysiology; Science Press: Beijing, 2003; pp 117-176. [武维华. 植物生理学. 北京: 科学出版社, 2003: 117-176.]

(42) Mohapatra, P. K.; Singh, N. R. Photosynth. Res. 2015, 123, 105. doi: 10.1007/s11120-014-0034-4

(43) Suzuki, H.; Tomita, O.; Higashi, M.; Abe, R. Catal. Sci. Technol. 2015, 5, 2640. doi: 10.1039/C5CY00128E

(44) Sasaki, Y.; Iwase, A.; Kato, H.; Kudo, A. J. Catal. 2008, 259, 133. doi: 10.1016/j.jcat.2008.07.017

(45) Miseki, Y.; Fujiyoshi, S.; Gunji, T.; Sayama, K. Catal. Sci. Technol. 2013, 3, 1750. doi: 10.1039/c3cy00055a

(46) Abe, R.; Shinmei, K.; Koumura, N.; Hara, K.; Ohtani, B. J. Am. Chem. Soc. 2013, 135, 16872. doi: 10.1021/ja4048637

(47) Sasaki, Y.; Kato, H.; Kudo, A. J. Am. Chem. Soc. 2013, 135, 5441. doi: 10.1021/ja400238r

(48) Maeda, K. ACS Catal. 2013, 3, 1486. doi: 10.1021/cs4002089

(49) Wan, K. J.; Adinaveen, T.; Vijaya, J. J.; Selvam, N. C. S. RSC Adv. 2016, 6, 10487. doi: 10.1039/C5RA24676H

(50) Zhang, L. J.; Li, S.; Liu, B. K.; Wang, D. J.; Xie, T. F. ACS Catal. 2014, 4, 3724. doi: 10.1021/cs500794j

(51) Sekizawa, K.; Maeda, K.; Domen, K.; Koike, K.; Ishitani, O J. Am. Chem. Soc. 2013, 135, 4596. doi: 10.1021/ja311541a

(52) Zhou, P.; Yu, J. G.; Jaroniec, M. Adv. Mater. 2014, 26, 4920. doi: 10.1002/adma.201400288

(53) Li, P.; Li, H. J.; Tu, W. G.; Zhou, Y.; Zou, Z. G. Acta Phys. Sin
2015, 64 (9), 094209. [李平, 李海金, 涂文广, 周 勇, 邹志 刚. 物理学报, 2015, 64 (9), 094209.]

(54) Abe, R. Bull. Chem. Soc. Jpn. 2011, 84 (10), 1000. doi: 10.1246/bcsj.20110132

(55) Yu, J. G.; Wang, S. H.; Low, J. X.; Xiao, W. Phys. Chem Chem. Phys. 2013, 15, 16883. doi: 10.1039/c3cp53131g

(56) Liao, W. J.; Murugananthan, M.; Zhang, Y. R. Phys. Chem. Chem. Phys. 2015, 17, 8877. doi: 10.1039/C5CP00639B

(57) Chen, X. X.; Huang, X. T.; Yi, Z. G. Chem. Eur. J. 2014, 20, 17590. doi: $10.1002 /$ chem. 201404284

(58) Yu, W. L.; Xu, D. F.; Peng, T. Y. J. Mater. Chem. A 2015, 3, 19936. doi: 10.1039/c5ta05503b

(59) Hong, Y. Z.; Jiang, Y. H.; Li, C. S.; Fan, W. Q.; Yan, X.; Yan, M.; Shi, W. D. Appl. Catal. B 2016, 180, 663. doi: 10.1016/j. apcatb.2015.06.057

(60) Yang, Y. X.; Guo, W.; Guo, Y. N.; Zhao, Y. H.; Yuan, X.; Guo, Y. H. J. Hazard. Mater. 2014, 271, 150. doi: 10.1016/j. jhazmat.2014.02.023

(61) Zhang, J. F.; Hu, Y. F.; Jiang, X. L.; Chen, S. F.; Meng, S. G.; Fu, X. L. J. Hazard. Mater. 2014, 280, 713. doi: 10.1016/j. jhazmat.2014.08.055

(62) Chen, X.; Tan, P. F.; Zhou, B. H.; Dong, H. G.; Pan, J.; Xiong, X. J. Alloy. Compd. 2015, 647, 456. doi: 10.1016/j. jallcom.2015.06.056

(63) Shi, L.; Liang, L.; Wang, F. X.; Liu, M. S.; Sun, J. M. J. Mater. Sci. 2015, 50, 1718. doi: 10.1007/s10853-014-8733-y

(64) Kumar, S.; Tonda, S.; Baruah, A.; Kumar, B.; Shanker, V. Dalton Trans. 2014, 43, 16105. doi: 10.1039/c4dt01076k

(65) Tian, N.; Huang, H. W.; He, Y.; Guo, Y. X.; Zhang, T. R.; Zhang, Y. H. Dalton Trans. 2015, 44, 4297. doi: 10.1039/ c4dt03905j

(66) Feng, Y.; Shen, J. C.; Cai, Q. F.; Yang, H.; Shen, Q. H. New J. Chem. 2015, 39, 1132. doi:10.1039/c4nj01433b

(67) He, Y. M.; Zhang, L. H.; Wang, X. X.; Wu, Y.; Lin, H. J.; Zhao, L. H.; Weng, W. Z.; Wan, H. L.; Fan, M. H. RSC Adv. 2014, 4 , 13610. doi: $10.1039 / \mathrm{c} 4 \mathrm{ra} 00693 \mathrm{c}$

(68) Bai, Y.; Wang, P. Q.; Liu, J. Y.; Liu, X. J. RSC Adv. 2014, 4, 19456. doi: $10.1039 / \mathrm{c} 4 \mathrm{ra} 01629 \mathrm{~g}$

(69) Meng, S. G.; Ning, X. F.; Zhang, T.; Chen, S. F.; Fu, X. L. Phys. Chem. Chem. Phys. 2015, 17, 11577. doi: 10.1039/ C5CP01523E

(70) Kondo, K.; Murakami, N.; Ye, C.; Tsubota, T.; Ohno, T. Appl. Catal. B 2013, 142, 362. doi: 10.1016/j.apcatb.2013.05.042

(71) Li, M. L.; Zhang, L. X.; Fan, X. Q.; Zhou, Y. J.; Wu, M. Y.; Shi, J. L. J. Mater. Chem. A 2015, 3, 5189. doi: 10.1039/ c4ta06295g

(72) He, Y. M.; Zhang, L. H.; Fan, M. H.; Wang, X. X.; Walbridge, M. L.; Nong, Q. Y.; Wu, Y.; Zhao, L. H. Sol. Energy Mater. Sol. Cells 2015, 137, 175. doi: 10.1016/j.solmat.2015.01.037

(73) He, Y. M.; Zhang, L. H.; Teng, B. T.; Fan, M. H. Environ. Sci. Technol. 2015, 49, 649. doi: 10.1021/es5046309 
(74) Li, W. B.; Feng, C.; Dai, S. Y.; Yue, J. G.; Hua, F. X.; Hou, H. Appl. Catal. B 2015, 168, 465.

(75) Yang, X. F.; Chen, Z. P.; Xu, J. S.; Tang, H.; Chen, K. M.; Jiang, Y. ACS Appl. Mater. Interfaces 2015, 7, 15285. doi: 10.1021/acsami.5b02649

(76) Zhao, G. X.; Huang, X. B.; Fina, F.; Zhang, G.; Irvine, J. T. S. Catal. Sci. Technol. 2015, 5, 3416. doi: 10.1039/c5cy00379b

(77) Zhu, J. F.; Zäch, M. Curr. Opin. Colloid Interface Sci. 2014, $14,260$.

(78) Chen, Z. H.; Wang, W. L.; Zhang, Z. G.; Fang, X. M. J. Phys. Chem. C 2013, 117, 19346. doi: 10.1021/jp406508y

(79) Wang, Q.; Li, Y. B.; Hisatomi, T.; Nakabayashi, M.; Shibata, N.; Kubota, J.; Domen, K. J. Catal. 2015, 328, 308. doi: 10.1016/j.jcat.2014.12.006

(80) Yamada, Y.; Nomura, A.; Tadokoro, H.; Fukuzumi, S. Catal. Sci. Technol. 2015, 5, 428. doi: 10.1039/C4CY01005A

(81) Lasa, H. I. D.; Rosales, B. S. Photocatalytic Technologies; Science Press: Beijing, 2010; pp 111-144; translated by Liu, Y., Liu, C. Y. [Lasa, H. I. D.; Rosales, B. S. 光催化技术. 刘 云, 刘春艳, 译. 北京: 科学出版社, 2010: 111-114.]

(82) Katsumata, H.; Tachi, Y.; Suzuki, T.; Kaneco, S. RSC Adv. 2014, 4, 21405. doi: 10.1039/c4ra02511c

(83) Yang, X. F.; Tang, H.; Xu, J. S.; Antonietti, M.; Shalom, M. ChemSusChem 2015, 8, 1350. doi: 10.1002/cssc.v8.8

(84) Tam, W. W. S.; Wong, T. W.; Wong, A. H. S. Atmos. Environ. 2015, 120, 360. doi: 10.1016/j.atmosenv.2015.08.068

(85) Rodopoulou, S.; Samoli, E.; Chalbot, M. C. G.; Kavouras, I. G. Sci. Total Environ. 2015, 536, 872. doi: 10.1016/j. scitotenv.2015.06.056

(86) Farhanian, D.; Haghighat, F. Build Environ. 2014, 72, 34. doi: 10.1016/j.buildenv.2013.10.014

(87) Yang, Z. Z.; Cai, T. J. Chin. J. Public Health 2003, 19 (6), 765 [杨振洲, 蔡同建. 中国公共卫生, 2003, 19 (6), 765.]

(88) Tang, Z. W.; Cheng, J. L.; Zhang, H, Y.; Fan, X. Y. J. Hydraul. Eng. 2009, 40 (9), 1064. [唐阵武, 程家丽, 张化永, 范修远. 水 利学报, 2009, 40 (9), 1064.]

(89) Chen, S. F.; Hu, Y. F.; Jiang, X. L.; Meng, S. G.; Fu, X. L. Mater. Chem. Phys. 2015, 149, 512.

(90) Wang, J. C.; Zhang, L.; Fang, W. X.; Ren, J.; Li, Y. Y.; Yao, H. C.; Wang, J. S.; Li, Z. J. ACS Appl. Mater. Interfaces 2015, 7, 8631. doi: 10.1021/acsami.5b00822

(91) Liu, Y. S.; Ji, G. B.; Dastageer, M. A.; Zhu, L.; Wang, J. Y.; Zhang, B.; Chang, X. F.; Gondal, M. A. $R S C A d v$. 2014, 4 , 56961. doi: 10.1039/C4RA10670A

(92) Sun, Y. H. Pet. Chem. Energy Cons. 2011, 2, 42. [孙延辉. 石油 和化工节能, 2011, 2, 42.]
(93) Ohno, T.; Murakami, N.; Koyanagi, T.; Yang, Y. J. $\mathrm{CO}_{2}$ Util. 2014, 6, 17.

(94) Shang, X. K.; Liu, R. J.; Zhang, G. J.; Zhang, S. J.; Cao, H. B.; Gu, Z. J. New J. Chem. 2014, 38, 1315. doi: 10.1039/ c3nj01184d

(95) Miseki, Y.; Kusama, H.; Sugihara, H.; Sayama, K. J. Phys. Chem. Lett. 2010, 1, 1196. doi: 10.1021/jz100233w

(96) Xu, Y.; Schoonen, M. Am. Mineral. 2000, 85, 543. doi: 10.2138/am-2000-0416

(97) Wang, H. Y.; Jiang, Z. P.; Yang, H. W. Res. Environ. Sci. 2008, 21 (5), 14. [王海燕, 蒋展鹏, 杨宏伟. 环境科学研究, 2008, 21 (5), 14.]

(98) Suzuki, T. M.; Iwase, A.; Tanaka, H.; Sato, S.; Kudo, A.; Morikawa, T. J. Mater. Chem. A 2015, 3, 13283. doi: 10.1039/ C5TA02045J

(99) Kato, H.; Sasaki, Y.; Shirakura, N.; Kudo, A. J. Mater. Chem. A 2013, 1, 12327. doi: 10.1039/c3ta12803b

(100) Zhou, H.; Ding, L.; Fan, T. X.; Ding, J.; Zhang, D.; Guo, Q. X. Appl. Catal. B 2014, 147, 221. doi: 10.1016/j. apcatb.2013.08.025

(101) Min, Y. L.; He, G, Q.; Xu, Q. J.; Chen, Y. C. J. Mater. Chem. A 2014, 2, 1294. doi: 10.1039/C3TA13687F

(102) Mogal, S. I.; Gandhi, V. G.; Mishra, M.; Tripathi, S.; Shripathi, T.; Joshi, P. A.; Shah, D. O. Ind. Eng. Chem. Res. 2014, 53, 5749. doi: 10.1021/ie404230q

(103) Katsumata, H.; Sakai, T.; Suzuki, T.; Kaneco, S. Ind. Eng. Chem. Res. 2014, 53, 8018. doi: 10.1021/ie5012036

(104) Zhu, Z. F.; Du, J.; Li, J. Q.; Zhang, Y. L.; Liu, D, G.; Yu, H. G. J. Funct. Mater. 2011, 42 (10), 1916. [朱振峰, 杜 娟, 李军 奇, 张艳丽, 刘佃光, 于红光. 功能材料, 2011, 42 (10), 1916.]

(105) Huang, Z. A.; Sun, Q.; Lv, K. L.; Zhang, Z. H.; Li, M.; Li, B. Appl. Catal. B 2015, 164, 420. doi: 10.1016/j. apcatb.2014.09.043

(106) Chen, S. H.; Peng, F.; Wang, H. J. Mod. Chem. Ind. 2004, 24 (7), 24. [陈水辉, 彭 峰, 王红娟. 现代化工, 2004, 24 (7), 24.]

(107) Modak, B.; Ghosh, S. K. J. Phys. Chem. B 2015, 119, 11089. doi: 10.1021/acs.jpcb.5b02906

(108) Yin, R.; Luo, Q. Z.; Wang, D. S.; Sun, H. T.; Li, Y. Y.; Li, X. Y.; An, J. J. Mater. Sci. 2014, 49, 6067. doi: 10.1007/s10853014-8330-0

(109) Zang, Y. P.; Li, L. P.; Li, X. G.; Lin, R.; Li, G. G. Chem. Eng. J. 2014, 246, 277. doi: 10.1016/j.cej.2014.02.068

(110) Wang, K.; Li, Q.; Liu, B. S.; Cheng, B.; Ho, W. K.; Yu, J. G. Appl. Catal. B 2015, 176, 44. doi: 10.1016/j. apcatb.2015.03.045 